\title{
propriétés hydrauliques et mécaniques des sols non saturés
}

\section{Pr. Silvan Andrei}

Laboratoire de Mécanique des Sols Université de Constantine et Institut de Construction Bucarest, Roumanie

\section{PROPRIETES HYDRAULIQUES ET MECANIQUES DES SOLS NON SATURES}

L'élément essentiel qui conditionne les propriétés des sols non saturés est l'existence d'une pression interstitielle négative (succion), due à l'interaction entre les trois phases constituant le sol, qui caractérise la capacité de rétention de ce corps hydrophile.

Dans la première partie de l'exposé sont présentés les résultats des recherches concernant la capacité de rétention de l'eau par les sols et notamment les méthodes utilisées pour établir la courbe de rétention, son expression analytique, les transferts d'énergie liés aux mouillages-séchages. I'utilisation de la courbe de rétention pour prévoir les effets des différents procédés d'assèchement.

La deuxième partie est consacrée à l'influence de la succion sur les mouvements de l'eau dans les sols non-saturés: I'infiltration, le drainage, l'ascen. sion capillaire, la migration de l'eau vers les racines des plantes, les méthodes pour prévoir la distribution d'équilibre de la teneur en eau sous les revêtements imperméables.

Pour les sols non saturés, pour lesquels il n'existe pas de correspondance univoque entre l'état de serrage et l'état d'humidité caractéristique, pour les sols saturés, les propriétés sont conditionnées en même temps par la porosité ainsi que par la teneur en eau. Pour cette raison, on présente dans la dernière partie de l'exposé la méthodologie pour l'étude de l'influence de ces deux paramètres sur les propriétés hydrauliques (succion, perméabilité) et mécaniques (résistance à la compression simple. résistance au cisaillement et paramètres $\Phi$ et c. module de déformation, etc.) des sols non saturés.

\section{HYDRAULIC AND MECHANICAL PROPERTIES OF NON SATURATED SOILS}

The essential element which influences the properties of non saturated soils is the existence of negative pore pressure (suction) due to the interaction between the three phases constituting the soil, which characterizes the retention capacity of this hydrophilous body.

In the first part of the report the author presents research results concerning the water retention capacity of soils and especially methods used to define the retention curve, its analytical expression, energy tranfers linked to wetting-drying, the use of the retention curve to forecast the effects of different drying processes.

The second part is devoted to the influence of suction on the movements of water in non-saturated soils: seepage, drainage, moisture rising by capillarity, migration of water towards the roots of plants, methods to forecast the equilibrium distribution of water content under waterproofing coating.

For non saturated soils for which there is no univocal correlation between the state of compaction and the state of humidity, characteristic for saturated soils, the properties are influenced at the same time by the porosity and the water content. For this reason the final section of the paper outlines the methodology for research on the influence of these two parameters on hydraulic properties (suction, permeability) and mechanical properties (compressive strength. shear strength and $\Phi$ and c parameters, deformation modulus, etc.) of non saturated soils. 
Comité Français de Mécanique des Sols 


\title{
propriétés hydrauliques et mécaniques des sols non saturés
}

\author{
par Pr. Silvan ANDREI
}

\section{INTRODUCTION}

Il existe beaucoup de régions où, en raison des conditions climatiques (arides, semi-arides, continentales extrêmes), les sols se trouvent la plupart du temps en état de non-saturation.

En même temps, on doit remarquer que la mécanique classique des sols s'est occupée principalement des sols saturés et que le comportement des sols non-saturés est moins bien connu.

C'est exactement pour cela qu'on a jugé utile de présenter cette étude sur les propriétés hydrauliques et mécaniques des sols non-saturés, à partir des résultats de recherches poursuivies pendant plusieurs années sur une gamme étendue de sols (fig. 1).

On doit mentionner que la plupart des résultats obtenus sont valables non seulement pour les sols mais aussi pour d'autres corps poreux hydrophiles : matériaux de construction (béton, briques, matériaux céramiques) et matériaux soumis au séchage (papier, bois, tourbe, charbon, etc.) [4].

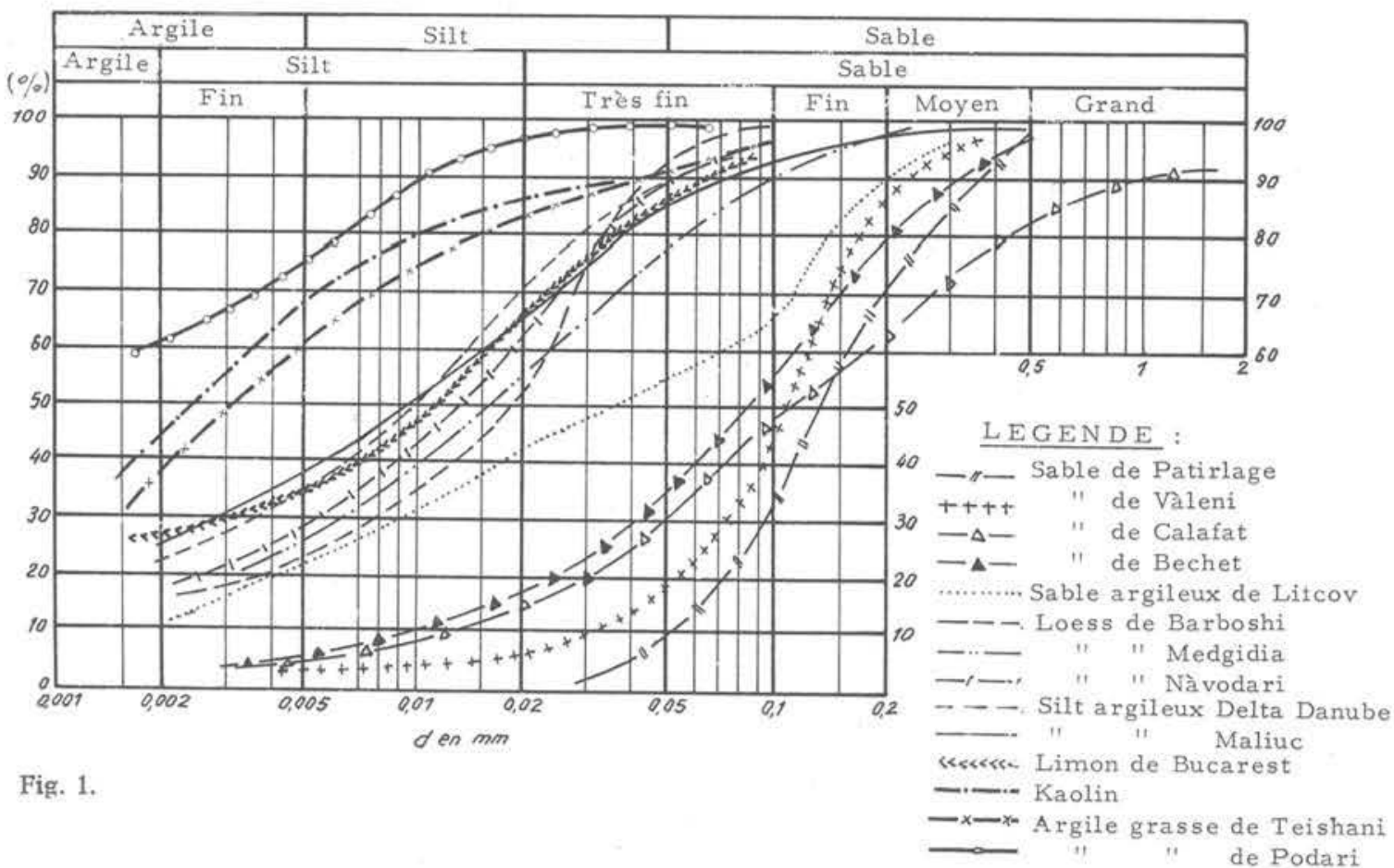

\section{ETAT DE NON SATURATION. MODE DE REPRESENTATION}

Généralement, on définit un sol comme saturé si les pores de la matrice constituant le squelette solide sont remplis par la phase liquide (l'eau).

Dans ce cas, il existe une correspondance univoque entre l'état de l'humidité et l'état de serrage, qui s'exprime par la relation :

$$
w_{\text {sat }}(\%)=\frac{n \rho_{w}}{(1-n) \rho_{s}} 100=e \frac{\rho_{w}}{\rho_{s}} 100=\rho_{w}\left(V_{100}-\frac{100}{\rho_{s}}\right)
$$

où

$w_{\text {sat }}$ est la teneur en eau pondérale ;

$n$, la porosité ;

$e$, l'indice des vides ; 
$\rho_{w}$ et $P_{s}$, les masses volumiques de l'eau et du squelette ;

$\mathrm{V}_{100}$, le volume correspondant à une masse de $100 \mathrm{~g}$ de la phase solide.

Puisque les forces d'interaction entre l'eau et le squelette minéral sont compensées, la pression interstitielle dans les sols saturés est généralement égale ou supérieure à la pression atmosphérique.

Les sols sont considérés comme non-saturés si dans leurs pores se trouve de l'air; ils sont alors des systèmes triphasiques (fig. 2).

Fig. 2.

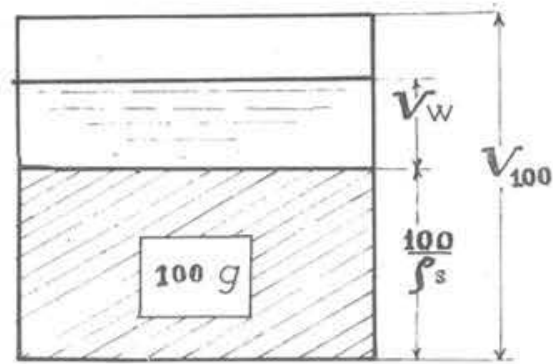

Dans ce cas, la correspondance univoque (1), mentionnée plus haut ne subsiste pas, c'est-à-dire qu'à une même porosité peuvent correspondre une infinité de teneurs en eau en fonction du degré de remplissage des pores par l'eau (degré de saturation, $\mathrm{S}_{r}$ ) ou qu'à une même teneur en eau le sol peut avoir différentes porosités.

Comme les propriétés des sols non-saturés sont déterminées par relations réciproques entre les phases constituantes, pour leur étude, on doit disposer d'un système de représentation permettant de suivre en même temps l'influence de l'état de serrage et de l'état d'humidité.

Parmi les systèmes qui ont été proposés, à notre avis le plus convenable est celui de Fernando Terracina [25] qui a construit un abaque ayant $w$ en abscisse et $V_{100}$ en ordonnée (fig. 3).

Dans ce cas, entre la masse volumique sèche $\rho_{d}$ et $V_{100}$ on a la relation :

$$
P_{d}=\frac{100}{V_{100}}
$$

On peut marquer en ordonnée des valeurs $\rho_{d}$ et tracer des droites horizontales correspondant à des chiffres ronds.

Sur l'abaque, on a représenté aussi les droites inclinées d'égale masse volumique humide $\rho$ correspondant à la relation :

$$
\rho=\rho_{d}\left(1+\frac{w}{100}\right)=\frac{100}{V_{100}}\left(1+\frac{w}{100}\right)
$$

D'après cette relation, on peut déduire que sur l'axe des ordonnées $(w=0) \rho$ et $\rho_{d}$ ont la même valeur.

D'après l'expression de la porosité :

$$
n=1-\frac{P_{d}}{P_{s}}=1-\frac{100}{V_{100}} \frac{1}{P_{s}}
$$

et l'indice des vides :

$$
e=\frac{n}{1-n}=\frac{\mathrm{V}_{100}}{100} \rho_{s}-1
$$

On en déduit que pour une certaine masse volumique des grains $\left(P_{s}\right)$ il existe des relations univoques entre $V_{100}$ et les valeurs $n$ et $e$. Sur cette base, on a tracé à la partie gauche de l'abaque, sur des droites correspondant aux masses volumiques des grains de sable $\left(P_{s}=2.65 \mathrm{~g} / \mathrm{cm}^{3}\right)$, silt $\left(P_{s}=2.70 \mathrm{~g} / \mathrm{cm}^{3}\right)$ ou argile $\left(\rho_{s}=2.72 \mathrm{~g} / \mathrm{cm}^{3}\right)$ les valeurs de $n$ et $e$ déduites en utilisant les relations (4) et (5).

Dans le cas des sols à pores remplis d'eau $\left(\mathrm{S}_{\mathrm{r}}=1\right)$, les teneurs en eau à saturation données par la relation (1) correspondent à une droite inclinée à $45^{\circ}$ par rapport aux axes et qui rencontre l'axe des ordonnées $(w=0)$ au point $V_{100}=\frac{\rho_{s}}{100}$; la position de ce point est donc déterminée par la masse volumique des grains.

En tenant compte de la définition du degré de saturation $\left(\mathrm{S}_{r}=\frac{w}{w_{\mathrm{sat}}}\right)$ et de la relation (1) on obtient :

$$
w=\mathrm{S}_{r} w_{\mathrm{sat}}=\mathrm{S}_{r} \rho_{w}\left(\mathrm{~V}_{100}-\frac{100}{\rho_{s}}\right)
$$

ou :

$$
\mathrm{V}_{100}=\frac{w}{\mathrm{~S}_{r} \rho_{w}}+\frac{100}{\rho_{s}}
$$

Faisant l'hypothèse que $P_{s}$ est constante, on obtient pour les valeurs rondes de $\mathrm{S}_{r}$ un faisceau de droites concourantes passant par le même point $\left(V_{100}=\frac{100}{P_{s}}\right)$ sur l'axe des ordonnées, qui se confond avec le point d'intersection de la droite de saturation avec cet axe. Sur l'abaque sont représentées en ligne interrompues les droites d'égales $\mathrm{S}_{r}(0.1,0.2,0.3$ jusqu'à 0.9$)$ correspondant à la valeur de la masse volumique des grains $P_{s}=2.68 \mathrm{~g} / \mathrm{cm}^{3}$.

Sur l'abaque, on peut représenter aussi la teneur en eau volumique exprimée comme le rapport entre le volume d'eau $V_{w}$ et le volume total $V_{100}$ :

$$
\theta=\frac{V_{w}}{V_{100}} 100=\frac{w \cdot 100}{P_{w} V_{100}}
$$

ce qui pour l'axe des abscisses $\mathrm{V}_{100}=100$ conduit au $\theta=w$; d'après la relation (7), pour $V_{100}=50$ on obtient $\theta=0.5 \mathrm{w}$ et on peut tracer ainsi les droites d'égale valeur $\theta$, et compléter de cette manière l'abaque proposée par Terracina [10].

La position de chaque point de l'abaque montre l'état d'humidité et de serrage du sol et est déterminée par deux des paramètres : $w, \mathrm{~S}_{r}, n$ ou $e, \rho_{d}$ et $\rho$; en utilisant l'abaque, les valeurs correspondantes des paramètres inconnus peuvent être facilement établies.

Par exemple, à un sable $\left(\rho_{s}=2.65 \mathrm{~g} / \mathrm{cm}^{3}\right)$ ayant $w=8 \%$ et $\rho=1.67 \mathrm{~g} / \mathrm{cm}^{3}$ correspond le point $\mathrm{P}$ de l'abaque, qui se trouve à l'intersection de la verticale menée par $b$ et de la droite inclinée d'égale valeur $\rho=1.67 \mathrm{~g} / \mathrm{cm}^{3}$ menée par $a$.

La pente de la droite $f$, qui lie le point $\mathrm{P}$ au point de l'axe des ordonnées correspondant à la densité des grains $\left(\rho_{s}\right)$, indique le degré de saturation du sol $\mathrm{S}_{r}=0.3$.

$\mathrm{Si}$ on mène par le point $\mathrm{P}$ une droite parallèle à l'axe des abscisses, on trouve $n=41.6 \%$ et $e=0.71$, sur les échelles de la porosité et de l'indice des vides, et à l'intersection avec la droite de la saturation, on trouve $w_{\text {sat }}=27 \%(c)$, qui correspond à $\rho=1.97 \mathrm{~g} / \mathrm{cm}^{3}(h)$; pour ce même point on trouve sur l'axe des ordonnées $V_{100}=64.5 \mathrm{~cm}^{3}$ (point $d$ ), respectivement $\rho_{d}=1.55 \mathrm{~g} / \mathrm{cm}^{3}$. De même on peut constater que la teneur en eau volumique correspondant au point P est $\theta=12.2 \%$.

Parmi les abaques proposés par différents auteurs, celle de F. Terracina présente certains avantages dus 

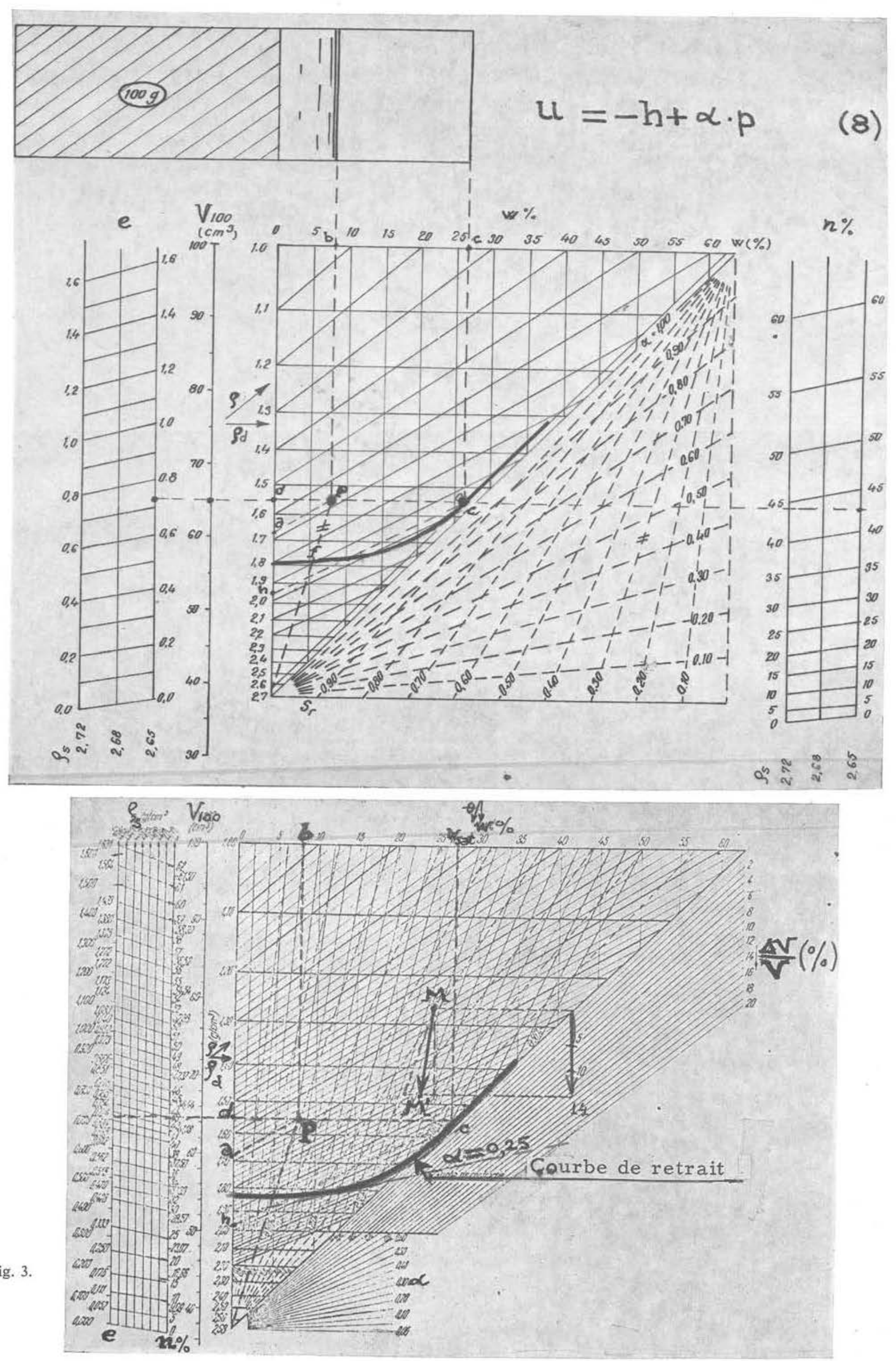


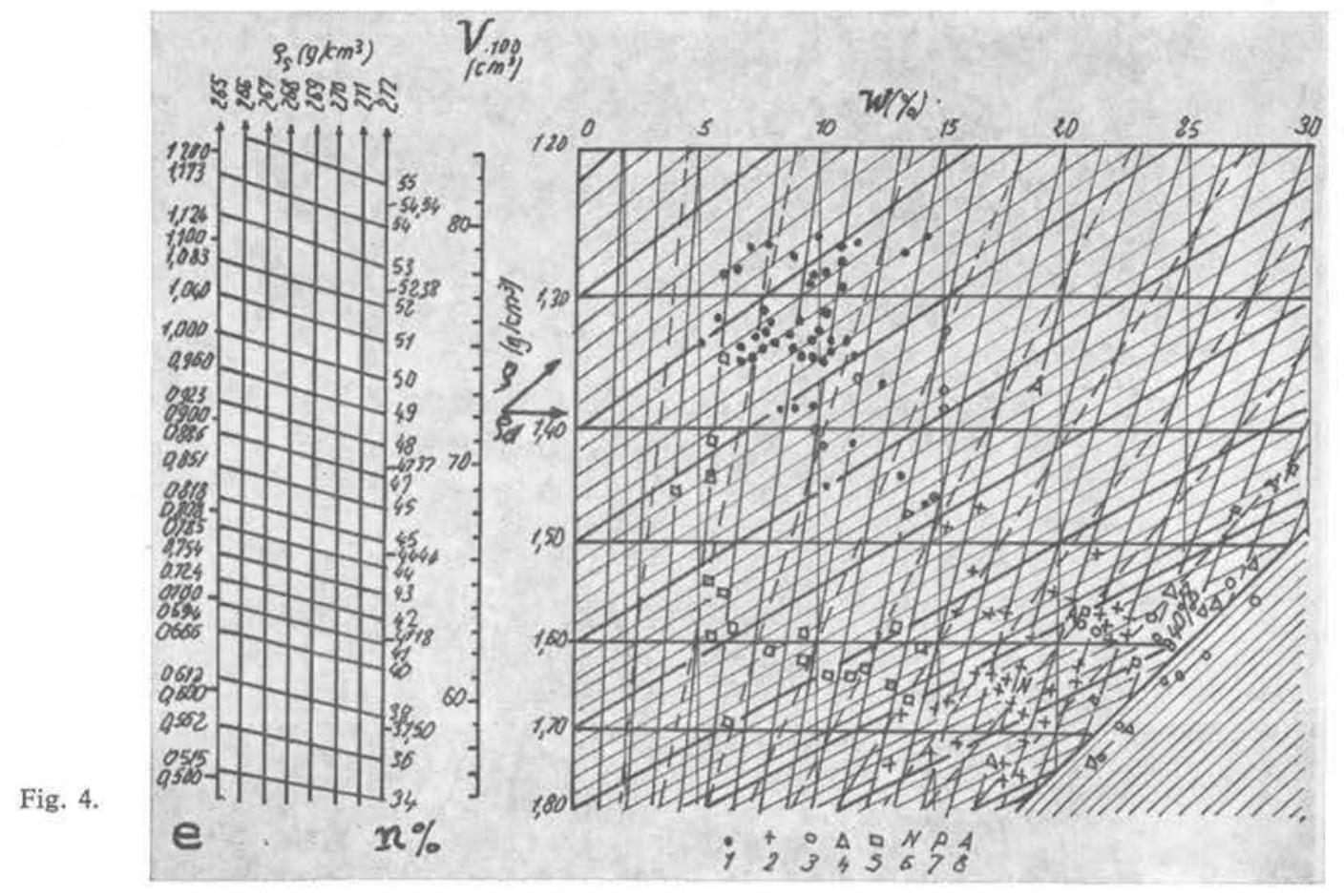

principalement à la linéarisation des courbes d'égales valeurs $w, \theta, \rho_{d}, \rho, V_{100}, \mathrm{~S}_{r}$. Par conséquent, l'abaque nous permet de suivre facilement les modifications de l'état d'humidité ou de serrage du sol sous l'action des différents facteurs naturels ou artificiels. Sur la figure 4 , on peut voir qu'à chaque sol naturel non-saturé de Roumanie correspond un certain " domaine d'existence " (loess de Baragan 1, limon de Bucarest 2, argile 3 , silt 4 , et sable 5 , de Ichalnita, ainsi que le sable argileux 6 , silt argileux 7 , et argile 8 , de la vallée du Danube) [5]. A partir des "domaines d'existences " des différents sols, établis à l'occasion des études antérieures, on peut par exemple prévoir les éventuelles pertubations de l'état dues à l'action de certains facteurs [6]. L'abaque donne aussi la possibilité de contrôler rapidement la correspondance entre les indices physiques établis par les essais de laboratoire et de détecter les éventuelles erreurs.

En Roumanie, l'abaque de Terracina a été utilisée il y a déjà de nombreuses années [5], [6], [24] A cette occasion, en dehors des propriétés remarquées par Terracina, on a mis en évidence d'autres possibilités de cet abaque. Ainsi, on peut représenter sur cet abaque la courbe de retrait $\left(V_{100}, w\right)$ d'un sol cohérent (fig. 3.): tant que le sol reste pratiquement saturé, la courbe de retrait est une ligne droite parallèle à la droite de saturation, inclinée à $45^{\circ}$ par rapport aux axes de coordonnées; au moment où l'air pénètre dans les pores, la droite devient une courbe à concavité tournée vers le haut qui s'éloigne de la droite de saturation et tend vers une droite parallèle à l'axe des abscisses pour les teneurs en eau réduites.

La détermination expérimentale de cette courbe par la méthode de la projection optique [4], [13] donne la possibilité de calculer le facteur de la compression $\alpha$, c'est-à-dire la pente de la courbe de retrait qui intervient dans l'expression de la pression interstitielle $u$ des sols non-saturés :

$$
u=-h+\alpha \cdot p
$$

où $h$ est la succion et $p$ la pression extérieure.

La valeur de $\alpha$ indique donc dans quelle mesure la pression appliquée $p$ est transmise à l'eau interstitielle. Ainsi, tant que le sol reste saturé $\alpha=1$, toute la pression est transmise à l'eau des pores, conclusion qui d'ailleurs est à la base de la théorie de la consolidation des sols cohérents saturés. Au fur et à mesure que le sol devient non-saturé $\alpha<1$, une partie seulement de la pression $p$ appliquée revient à l'eau interstitielle, l'autre partie $(1-\alpha)$ se transmettant au squelette du sol. Enfin, dans le domaine des petites teneurs en eau, le sol ne présentant pas de variations de volume $\left(d V_{100}=0\right)$, la courbe de retrait est horizontale $\Delta \mathrm{V}_{100}=0$, c'est-à-dire que toute la pression revient au squelette. Pour faciliter la détermination du facteur de compression, dans la partie inférieure de l'abaque, existe un faisceau de droites d'égale valeur de $\alpha$. L'abaque donne aussi la possibilité de suivre les variations du volume $\left(\frac{\Delta V_{100}}{V_{100}}\right) 100$. Dans ce but, sous la droite de saturation on trace les droites correspondant aux différents pourcentages de la variation du volume [10]. Ainsi, on peut, par exemple, suivre facilement les modifications de l'état de serrage pendant l'essai oedométrique. Par exemple, entre l'état initial $(M)$ et l'état final $\left(M^{\prime}\right)$ correspond une modification de volume de $14 \%$.

On doit remarquer que, pour l'étude systématique des sols non-saturés, l'abaque ouvre des possibilités qui jusqu'à présent n'ont pas été entièrement exploitées. 


\section{INTERACTION ENTRE LES PHASES CONSTITUANTES ET LES PROPRIETES DU SOL}

L'élément essentiel qui conditionne les propriétés des sols non-saturés est l'existence d'une pression interstitielle négative (succion), due à l'interaction entre les trois phases constituant le sol, qui caractérise la capacité de rétention des corps hydrophiles. Pour comprendre, donc, la capacité de rétention de l'eau dans les sols, on doit analyser un peu les phénomènes d'interaction.

La capacité de rétention des sols sableux est déterminée par le déficit de pression (succion au-dessous) des ménisques capillaires (fig. 5). D'après la loi de Laplace, plus le rayon $(r)$ du ménisque est petit (échelle des dimensions, fig. 6), plus la succion est grande (échelle des énergies, fig. 7) [3].

$$
h=\frac{0.15}{r}
$$

La capacité de rétention des sols argileux est déterminée par les propriétés d'adsorption de la paillette d'argile. Les théories les plus modernes conduisent à représenter les paillettes d'argile avec des charges négatives sur les grandes faces et des charges positives sur les petites (fig. 8). Certains ions sont attirés et retenus à proximité immédiate de la paillette en constituant une couche de contre-ions; par le jeu de mêmes charges électriques, d'autres ions hydratés sont maintenus à certaines distances par rapport à la paillette constituant ce que l'on nomme la couche diffuse. C'est ainsi que l'on explique [4] la présence à proximité de la paillette d'une couche d'eau adsorbée,

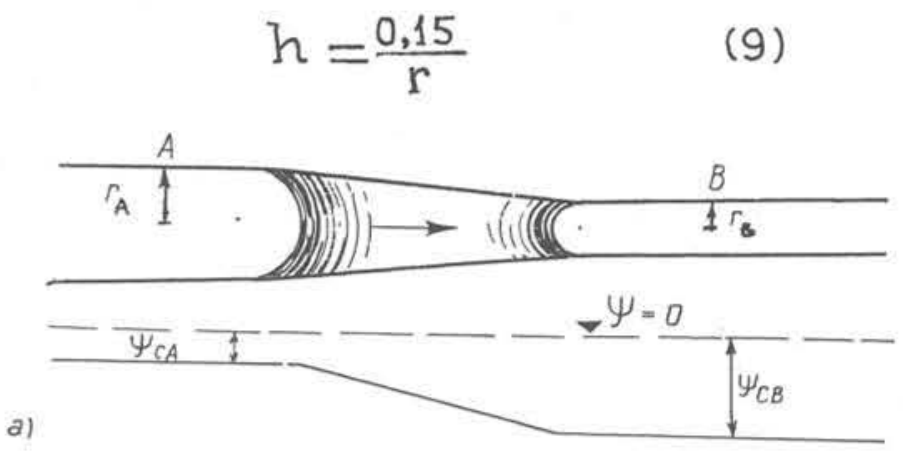

b)

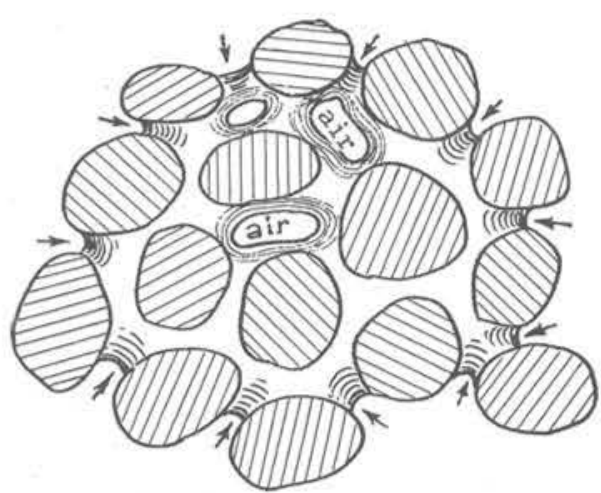

Fig. 5.

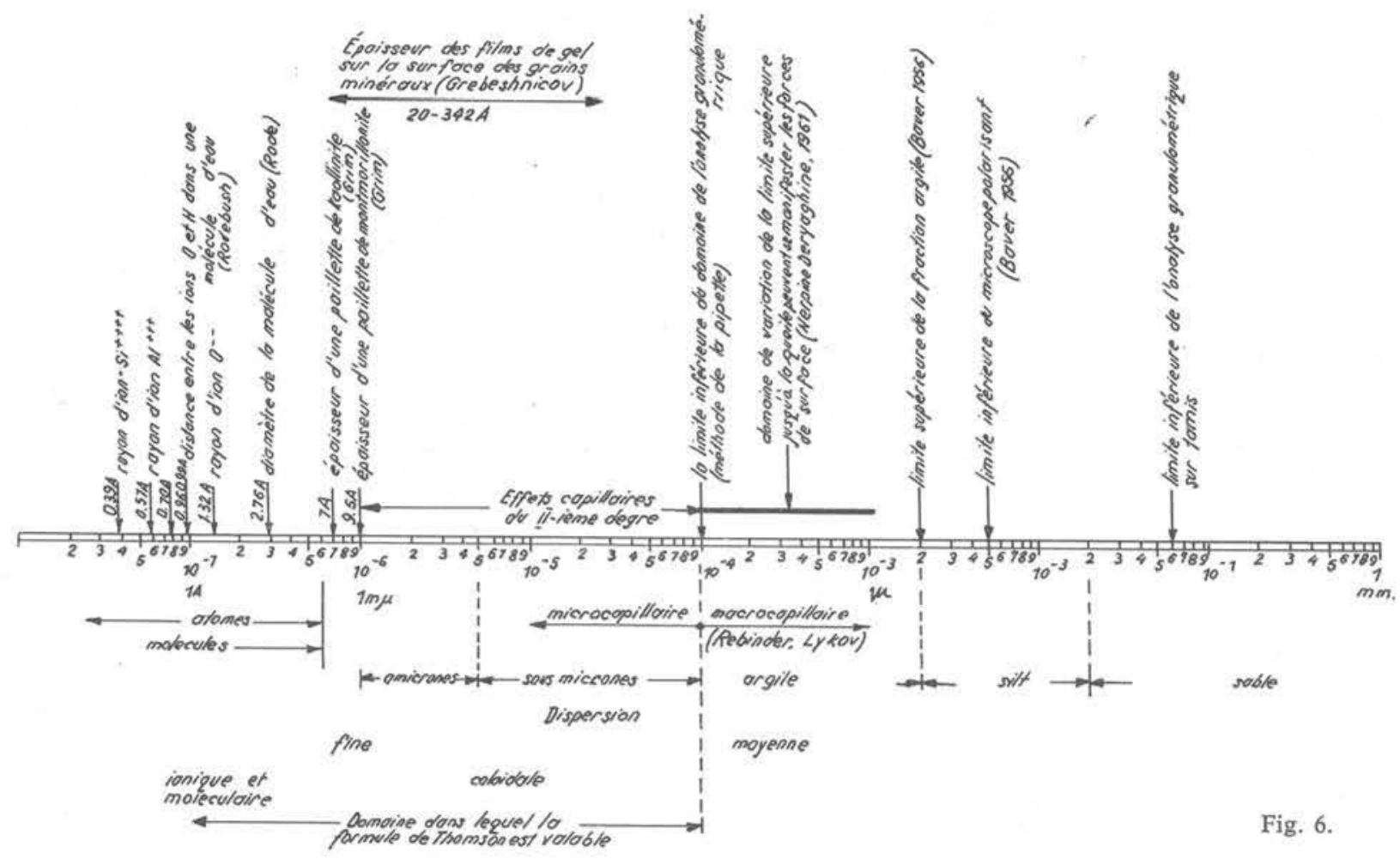

constituée par une auréole d'ions et de molécules dipôlaires d'eau; plus la distance des ions ou dipôles par rapport à la paillette est petite, plus les forces qui conduisent à leur rétention sont grandes et empêchent leurs mouvements cinétiques. Par conséquent, la liberté de mouvement des constituants de la couche d'eau adsorbée est plus ou moins restreinte par comparaison à celle de l'eau libre; de ce point de vue, les dipôles d'eau adsorbée ont une situation intermédiaire entre ceux de l'eau liquide et ceux de la glace. On dit que l'énergie libre de l'eau adsorbée est plus petite que celle de l'eau libre. La diminution de l'énergie libre se 
DOMAINE OES METHOOES POUR LA OETERHIMETION OE LA SULCION

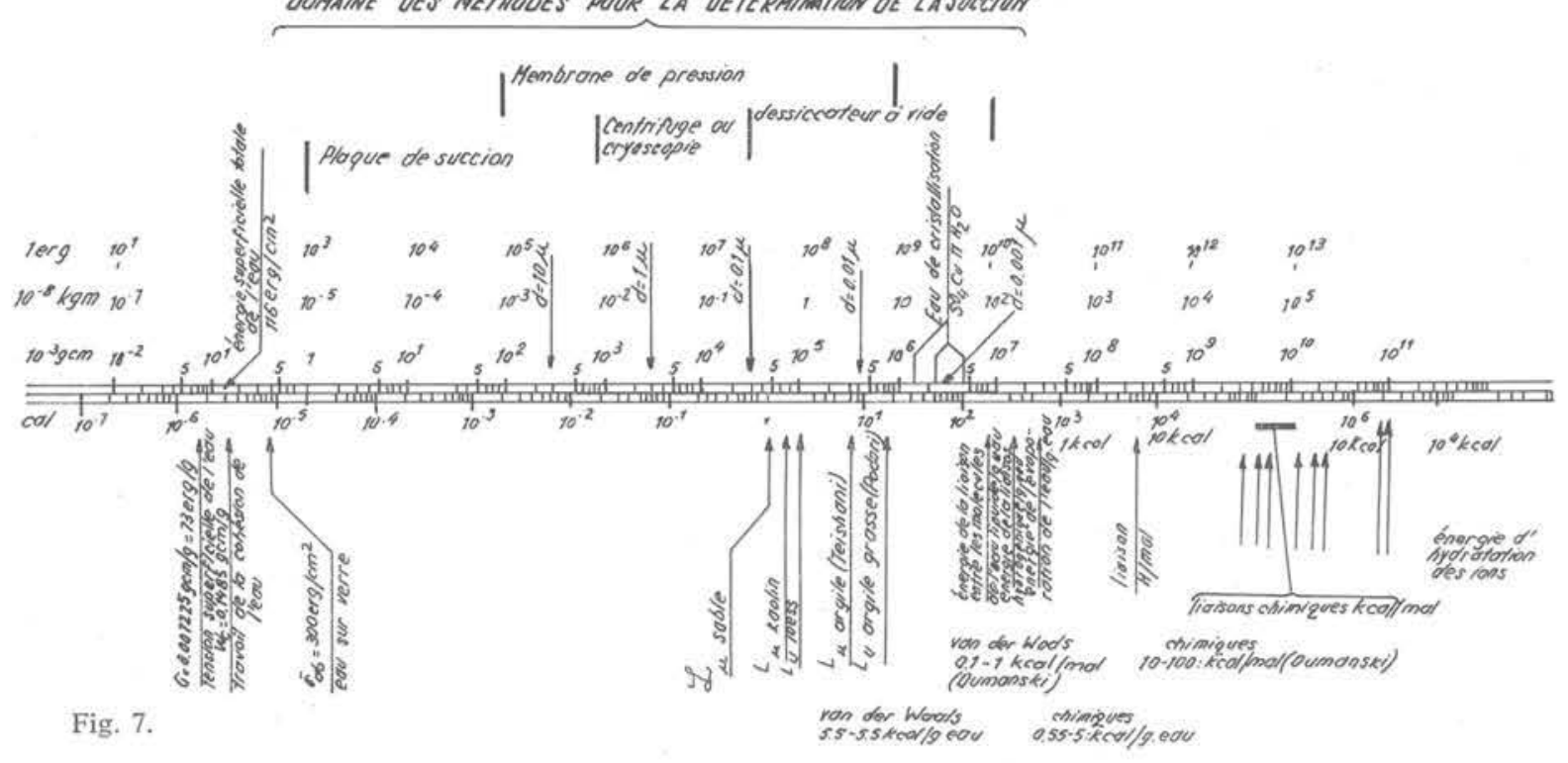

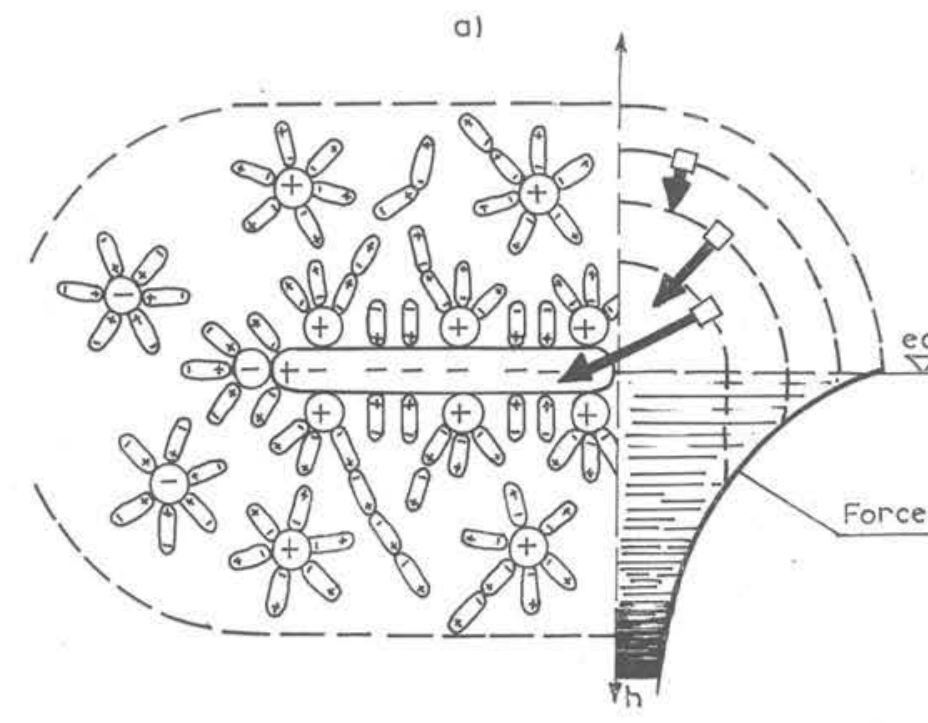

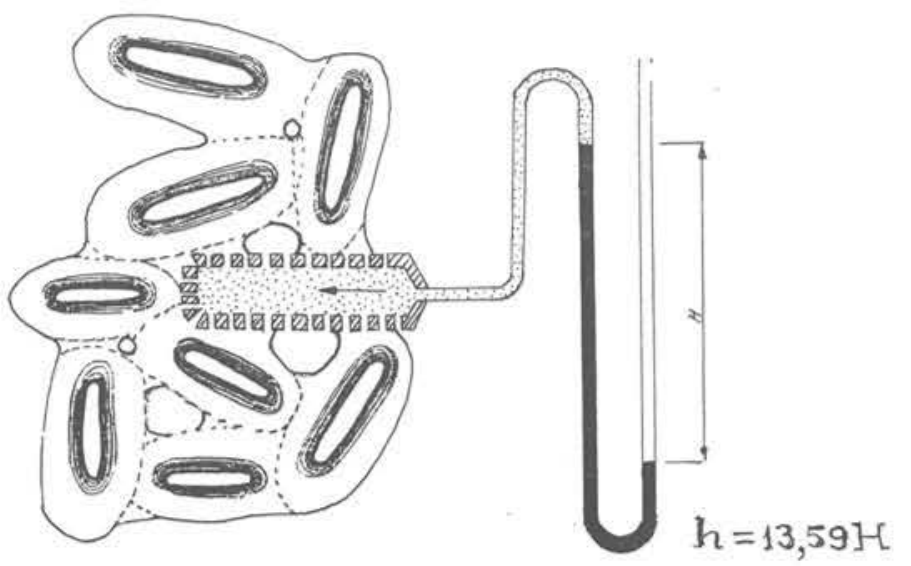

Fig. 8 . traduit par la transformation d'une partie d'énergie cinétique des molécules d'eau en chaleur de mouillage.

A la périphérie de l'eau adsorbée, il existe un échange continu entre les ions et les dipôles de la couche diffuse et ceux de l'eau libre, c'est-à-dire un équilibre dynamique.

L'épaisseur de la couche d'eau adsorbée dépend dans une large mesure de la nature et de la charge eau libre

électrique des ions. Plus ces charges sont grandes, plus la charge électrique totale de la paillette est compensée par un nombre moins important d'ions; par conséquent, l'épaisseur de la couche d'eau adsorbée est plus réduite.

Par exemple, les couches constituées avec des ions monovalents $\left(\mathrm{Li}^{+}, \mathrm{Na}^{+}, \mathrm{K}^{+}\right)$sont plus épaisses que celles des ions bivalents $\left(\mathrm{Ca}^{2+}, \mathrm{Mg}^{2+}\right)$ et beaucoup plus que celles des ions trivalents $\left(\mathrm{AI}^{3+}, \mathrm{Fe}^{3+}\right)$. L'intensité des forces avec lesquelles les ions et les dipôles d'eau sont attirés s'accroît rapidement avec la diminution de leurs distances par rapport à la surface de la paillette. A proximité immédiate de cette surface, l'eau est soumise à des pressions énormes (environ 10000 bars) qui modifient des propriétés en les approchant de celles d'un solide.

Comme montré ci-dessus, plus la molécule d'eau se trouve à proximité de la paillette, plus les forces de liaisons sont grandes, sa mobilité est réduite et son énergie potentielle est diminuée par rapport à celle de l'eau libre (fig. 8). Par conséquent, si on met en contact l'eau libre avec une couche d'eau adsorbée, il y aura migration d'eau vers la paillette (fig. 9) qui a tendance à compléter sa couche d'eau adsorbée. 
a)

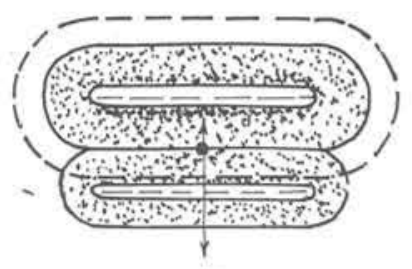

b).

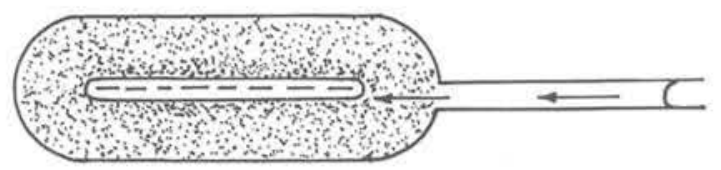

c).

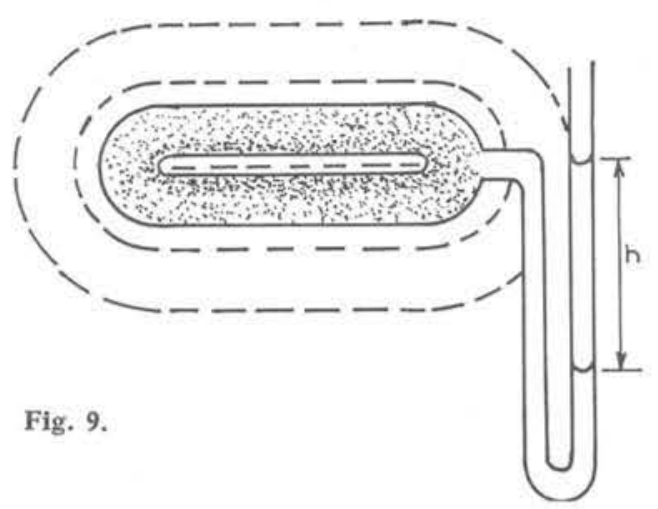

La paillette ayant une couche incomplète va exercer une succion sur l'eau libre (fig. $9 \mathrm{~b}$ ); par conséquent, dans les sols non-saturés - ayant les courbes adsorbées incomplètes - existe un déficit de pression par rapport à la pression atmosphérique, c'est-à-dire une succion. Plus l'épaisseur de la couche est petite par rapport à l'épaisseur maximale possible, c'est-à-dire plus la teneur en eau est petite, plus la succion qui se manifeste à la périphérie de la couche sera grande (fig. 9 c).

Fig. 10.

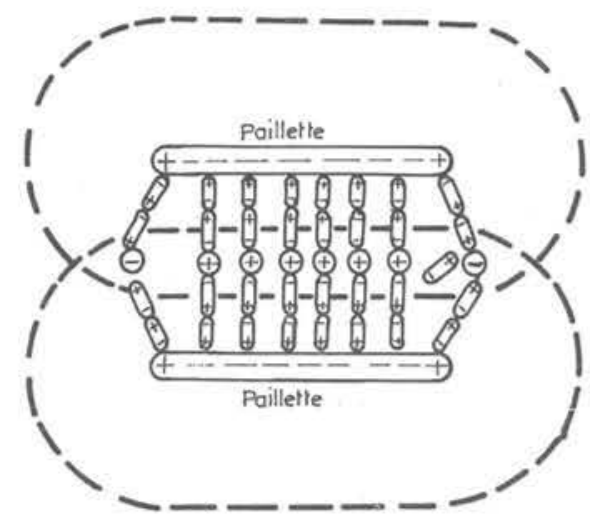

De même, l'existence du complexe d'adsorption enveloppant la paillette argileuse facilite l'établissement des ponts de liaison constitués par les ions, et les dipôles d'eau qui imprime aux sols fins une certaines cohésion (fig. 10). A cause de leur cohésion, les sols argileux ont la propriété de résister à des contraintes de compression, traction ou cisaillement ; la réalisation de ces ponts de liaison est en même temps à la base de processus de floculation ou coagulation tandis que leur destruction s'appelle défloculation ou peptisation. a).

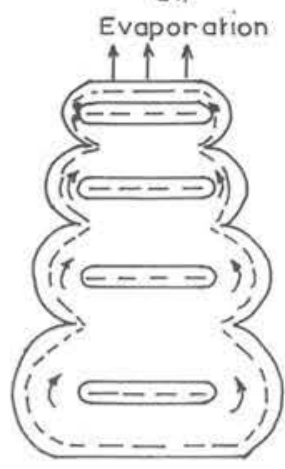

Fig. 11.

Rerraik b).

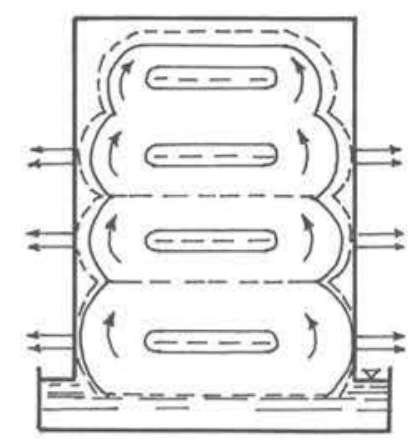

Gonflement

Le retrait et le gonflement, sont des conséquences de la modification de l'épaisseur des couches d'eau adsorbée (fig. 11) et du réarrangement des particules d'argile.

D'une manière analogue, on peut expliquer les autres propriétés des sols. Par exemple, les sols ayant de grosses couches d'eau adsorbée présentent de minces sections de pores occupées par l'eau libre (fig. 12 a) et, par conséquent, présentent de faibles perméabilités. Dans les argiles actives, ayant des porosités très réduites, le mouvement de l'eau peut commencer seulement si le gradient hydraulique dépasse une certaine valeur $i_{o}-$ gradient hydraulique initial - suffisante pour débloquer les bouchons d'eau adsorbée qui obturent les pores (fig. 12) [4].
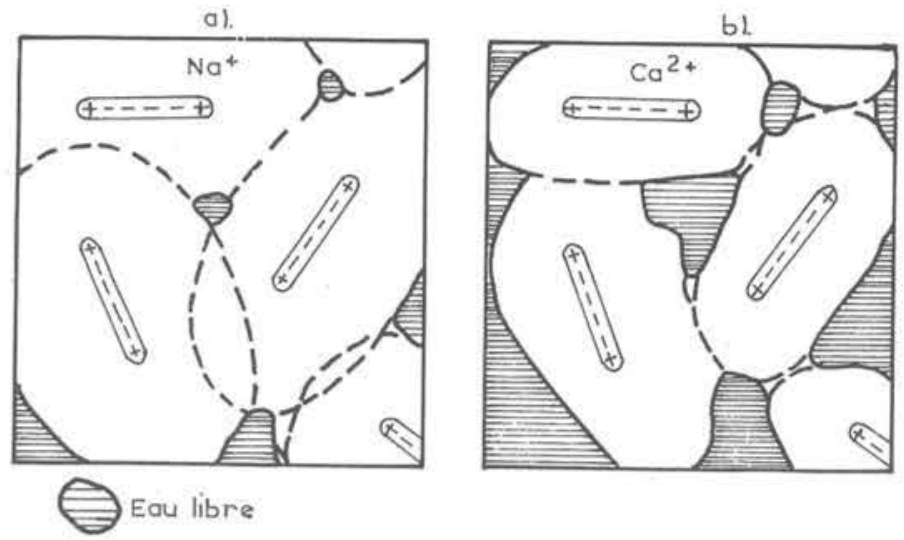

Fig. 12.

D'une manière similaire, on peut expliquer les effets du gradient thermique ou électrique sur les propriétés du sol.

Tout ce que l'on a montré plus haut témoigne que seule la connaissance des phénomènes d'interaction entre les phases constituant le sol donne la possibilité d'expliquer ses propriétés et de prévoir son comportement sous l'action des contraintes.

Même si les images concernant la constitution des sols sableux (fig. 5) ou argileux (fig. 8) restent encore un peu trop schématisées, leur utilisation s'avère nécessaire pour une meilleure compréhension des propriétés des sols. 


\section{RECHERCHES CONCERNANT LES PROPRIETES HYDRAULIQUES DES SOLS}

Les propriétés hydrauliques des sols non-saturés sont déterminées par la capacité de rétention de la matrice du squelette minéral. Pour caractériser l'intensité de rétention de l'eau par le squelette solide des matériaux poreux, on emploie différentes méthodes s'adressant à certains phénomènes physiques en relation avec l'interaction de l'eau et de la matrice solide, à avoir : le dégagement de chaleur due au mouillage (méthode de la chaleur de mouillage) (fig. 13), l'adsorption de l'eau d'une atmosphère ayant une humidité relative bien déterminée jusqu'à la réalisation de l'équilibre de la pression des vapeurs (méthodes des isothermes de sorption-désorption), (fig. 14), le drainage de l'eau des matériaux poreux par l'application d'une différence de pression (méthode de la succion) [3].

Les résultats représentés sur les figures 13 et 14 montrent que les courbes chaleur de mouillage-teneur en eau et les isothermes de sorption permettent de bien différencier l'activité du sol par rapport à l'eau. De même, les deux premières méthodes permettent de déduire la surface spécifique des particules du sol, ainsi que de caractériser la capacité de rétention dans le domaine hydroscopique, c'est-à-dire pour des teneurs en eau allant jusqu'à la teneur en eau d'hygroscopicité maximale $\left(w_{\mathrm{HM}}\right)$. Dans le domaine hygroscopique, les échanges d'humidité entre le corps poreux hydrophile et l'atmosphère ambiante se font seulement sous forme de vapeur d'eau.
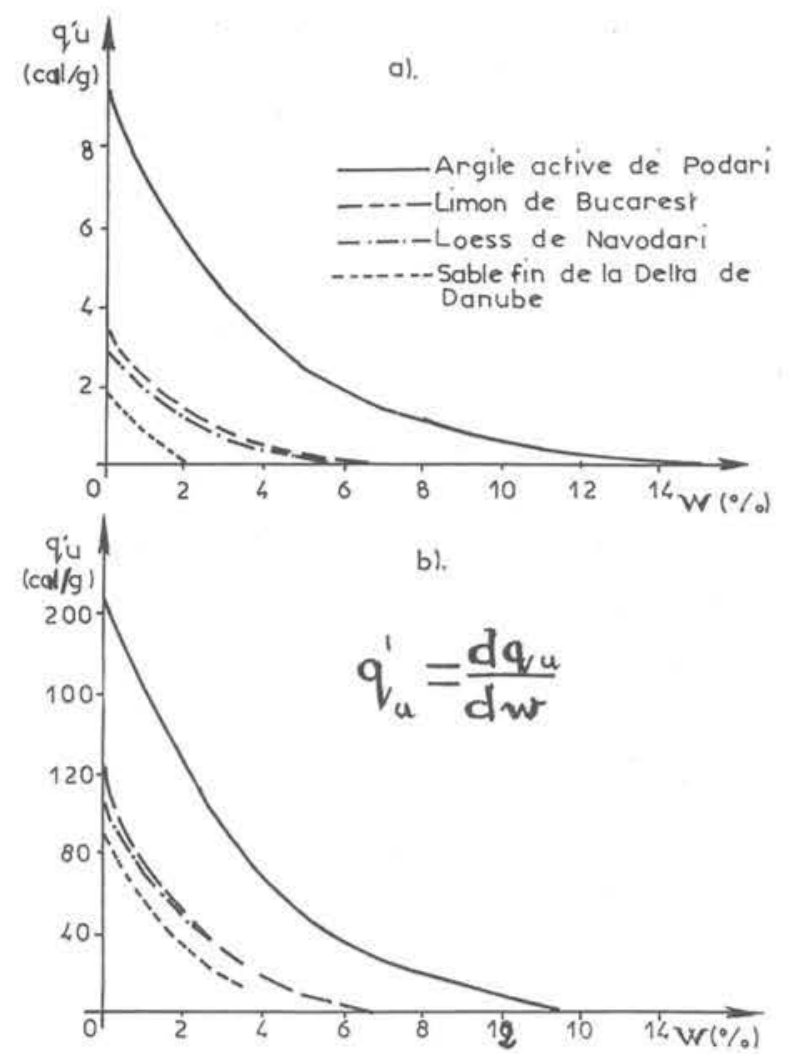

Fig. 13.

Si on a besoin de caractériser la capacité de rétention dans tout le domaine des teneurs en eau possibles, on doit faire appel à la courbe succion-teneur en eau. La succion $h$ représente le déficit de pression par rapport à la pression atmosphérique qui apparaît dans
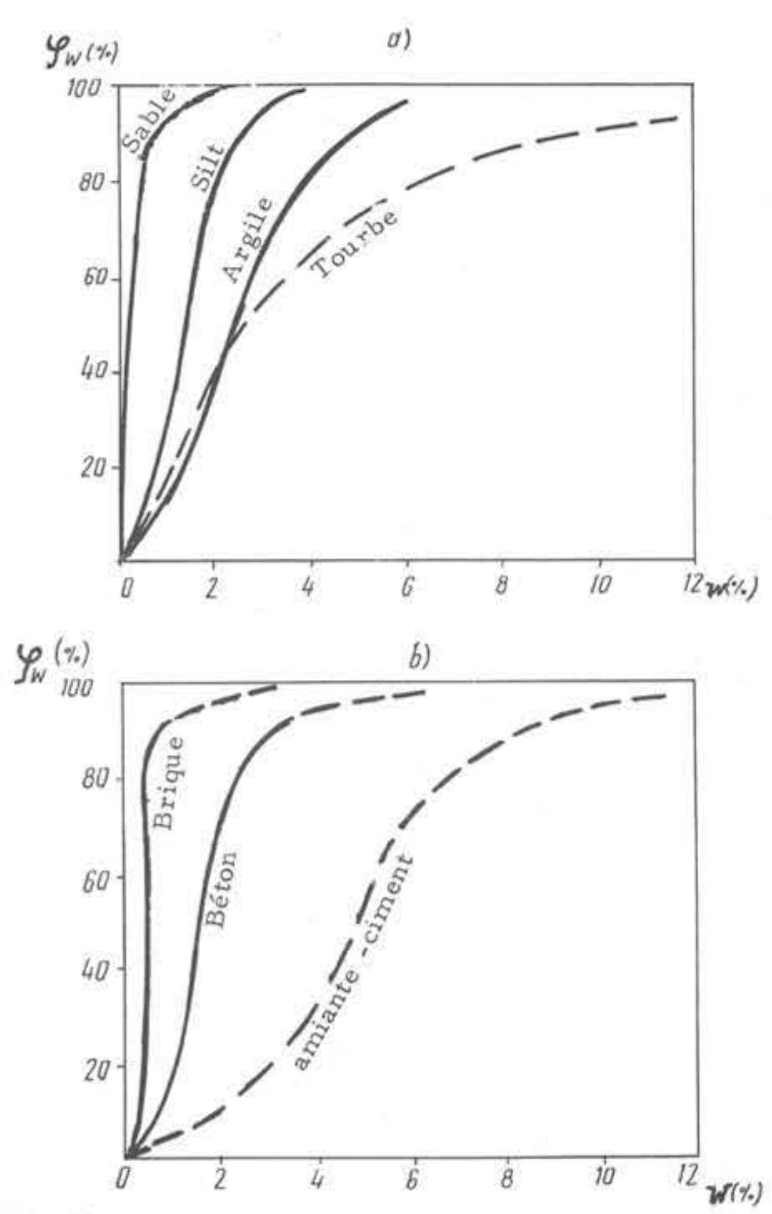

Fig. 14.

les pores d'un matériau poreux hydrophile à la suite des forces de liaison entre l'eau et le squelette solide ; dans un sable non-saturé, ces forces sont de nature capillaire (fig. 5), alors que dans le cas des sols argileux, l'effet des forces d'adsorption prédomine (fig. 8). Etant donné que les succions varient dans une gamme très large, de zéro pour le sol saturé, jusqu'à 10000 bars ( $h=10^{7} \mathrm{~cm}$ colonne d'eau), pour le sol séché à l'étuve, Schofield (en 1935), cité par Croney (1952) [13] a proposé qu'on se serve du symbole $p F$ (l'indice sorptionnel), qui représente le logarithme décimal de la succion, exprimé en centimètre colonne d'eau :

$$
p \mathrm{~F}=\log h
$$

La courbe succion-teneur en eau donne la possibilité d'établir la quantité d'eau retenue pour différentes succion (fig. 15).

Chaque changement qui concerne l'interaction entre les phases constituant le sol se fait sentir sur sa capacité de rétention [3]. Plus le sol est fin, c'est-àdire plus l'intensité des forces d'interaction est grande plus la succion de l'eau est grande (fig. 15 a). D'après la figure $15 \mathrm{~b}$, on peut remarquer qu'une petite augmentation de la fraction fine (silt) dans un mélange de sable fin (NV) et silt agrandit considérablement la capacité de rétention du mélange. Les essais menés par Road Research Laboratory ont démontré que la capacité de rétention de la montmorillonite est presque deux fois plus grande que celle de la kaolinite 


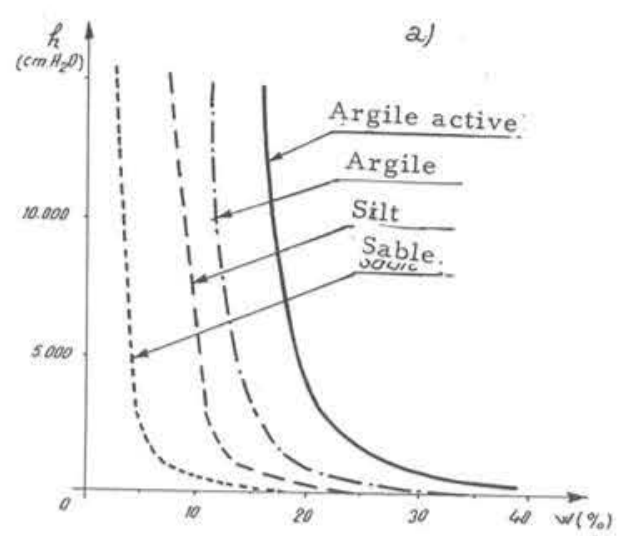

Fig. 15.

b)

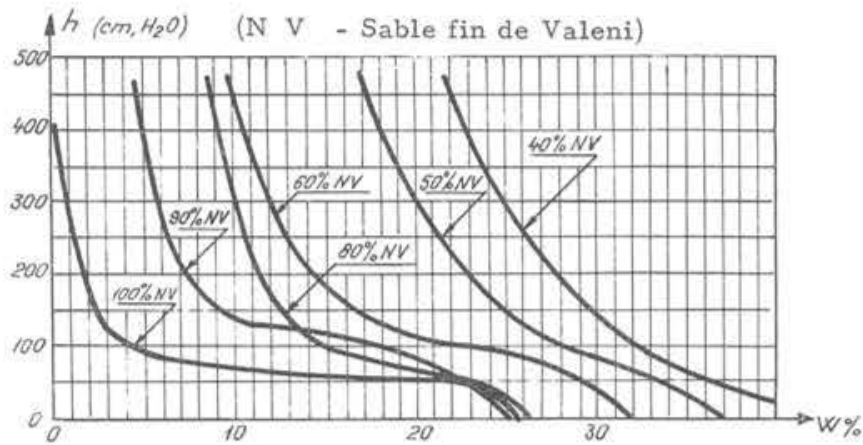

(fig. 16 a) [14]. D'après la figure $16 \mathrm{~b}$ où est représentée l'influence de l'état de serrage et d'humidité sur la succion exprimée à l'aide de l'indice sorptionnel pour un sable, un silt et une argile, on peut déduire que l'effet des modifications structurales (densité) se fait sentir spécialement dans les domaines des petites succions [3]. On doit remarquer en même temps que les argiles actives restent saturées même pour des succions assez grandes ( $p \mathrm{~F} \mathrm{4}$, c'est-àdire 10 bars).

La connaissance de la courbe de rétention d'un sol nous permet d'évaluer aussi les transferts d'énergie liés aux processus de drainage ou humidification.
Ainsi, si l'on tient compte que pour des conditions isothermes, la succion représente un potentiel négatif (fig. 17) on peut déduire que pour transformer une quantité élémentaire d'eau $d w$, retenue avec la succion $h$, en eau libre, le milieu extérieur doit dépenser, pour chaque gramme de $\mathrm{sol} \mathrm{sec}$, un travail spécifique de drainage [2] :

$$
d \varrho=h \cdot d w
$$

correspondant à la bande hachurée (fig. 17 a).

Lorsque la teneur en eau subit un changement de $w_{1}$ à $w_{2}$, le travail spécifique sera :

$$
\mathcal{L}=\int_{w_{1}}^{w_{2}} h d w
$$

qui a comme correspondance graphique l'aire $\mathrm{BC} \mathrm{C}^{\prime} \mathrm{B}$ (fig. $17 \mathrm{~b}$ ).

Si l'on suit l'évaluation dans le temps du processus, on doit introduire la notion de puissance spécifique de drainage ou de mouillage :

$$
\mathscr{Q}=\frac{d \mathscr{L}}{d t}
$$

\section{a)}

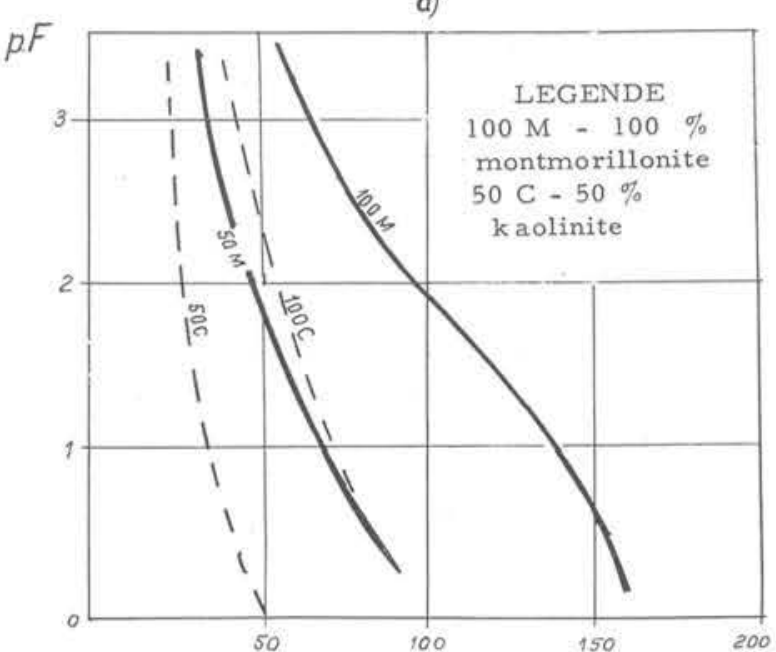

$w(\%)$

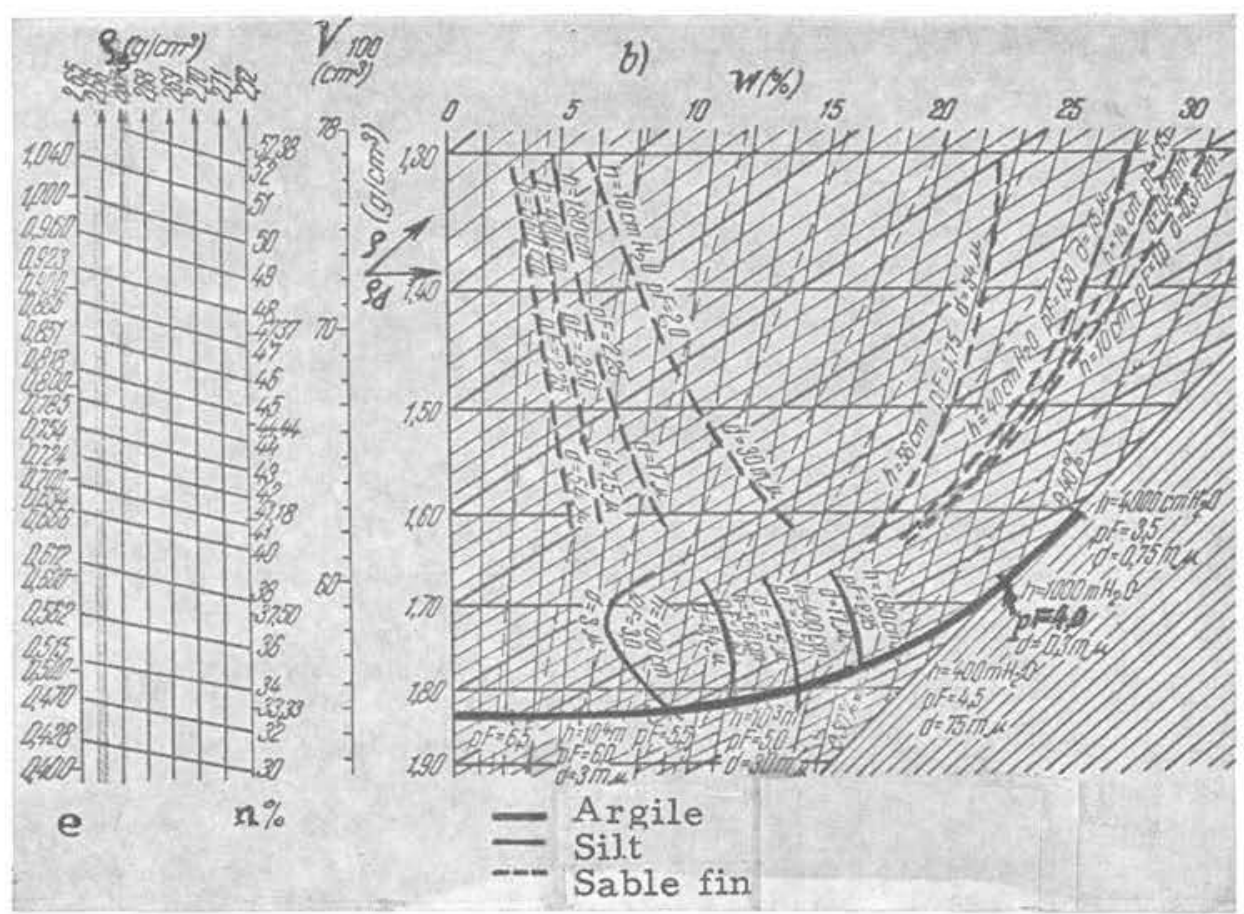



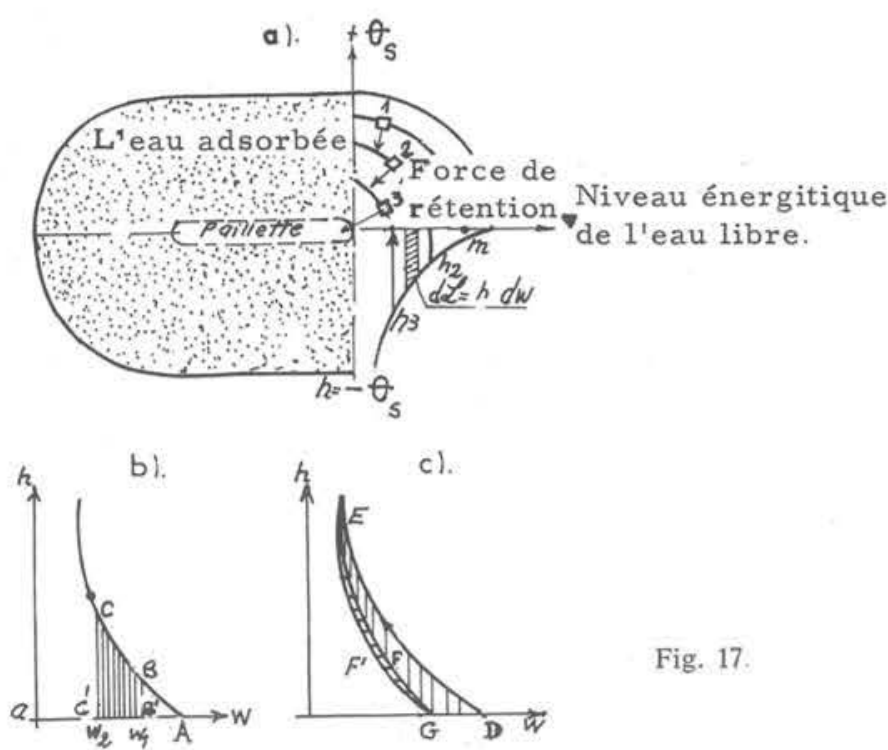

Si l'on analyse un cycle de drainage-mouillage, comme celui de la figure 17 , dans le sens des notions introduites auparavant, on trouve que la surface de la boucle d'hystérésis (GFEF'G) se trouvant entre les branches de drainage et de mouillage représente la quantité même d'énergie transformée irréversiblement au cours du processus, c'est-à-dire :

$$
\Delta \ell=f_{d r}-f_{u}
$$

où $\mathcal{E}_{d r}$ est le travail spécifique de drainage et $\mathcal{E}_{u}$ est le travail spécifique de mouillage.

Il en résulte donc, que pour effectuer un processus de drainage avec une vitesse donnée, il faut dépenser une énergie qui sera restituée seulement en partie au cours du processus inverse de mouillage, ce qui correspond d'ailleurs pleinement au deuxième principe de la thermodynamique. D'après ce principe, plus la vitesse du développement des processus est grande, autrement dit, plus les processus réels s'éloignent de ceux quasi-statiques, plus la cote irréversible de l'énergie sera grande et, par conséquent, la surface de la boucle d'hystérésis augmentera.

Les résultats expérimentaux montrent que, plus le cycle des variations de la succion comprend une gamme étendue, plus l'énergie dépensée irréversiblement au cours de processus et représentée par la surface de la boucle d'hystérésis sera grande. Ainsi, par exemple, dans le cas d'une argile grasse, lorsque la succion varie dans le domaine $p \mathrm{~F} 1 \div p \mathrm{~F} 3$ ( $h=10 \div 316 \mathrm{~cm} \mathrm{H}_{2} \mathrm{O}$ ) l'énergie dépensée n'est que de $3.75 \mathrm{~g} \mathrm{~cm} / \mathrm{g}$, tandis que pour le domaine de variation $p \mathrm{~F} 1$ à $p \mathrm{~F} 3.9\left(h=10\right.$ à $\left.794 \mathrm{~cm} \mathrm{H} \mathrm{H}_{2} \mathrm{O}\right)$, la dépense d'énergie a augmenté jusqu'à $12.50 \mathrm{~g} \mathrm{~cm} / \mathrm{g}$, c'est-à-dire de 3.3 fois, pour atteindre $6700 \mathrm{~g} \mathrm{~cm} / \mathrm{g}$ pour le domaine de variation $p \mathrm{~F} 1$ à $p \mathrm{~F} 7$.

De l'interprétation graphique du travail spécifique de drainage et de mouillage, il résulte aussi que la surface hachurée DEFG de la figure $17 \mathrm{c}$, comprise entre les deux branches de drainage d'une argile (branches qui partent de points différents sur l'axe des teneurs en eau et qui se rencontrent en un même point), peut être interprétée comme représentant la valeur du travail dépensé pour l'arrangement des particules du sol de façon plus serrée. En effet, pour les matériaux à structure rigide, dans le cas de répétition des cycles drainage-mouillage, on obtient les mêmes branches de courbe, c'est-à-dire qu'aucune énergie n'est dépensée pour replacer les particules du sol d'une manière plus compacte.

L'introduction des notions définies plus haut s'avère utile pour une meilleure compréhension des phénomènes de drainage-mouillage ainsi que pour établir des corrélations à la base énergétique entre différents indices hydrauliques des sols [3].

Ainsi, à partir des courbes de rétention $(h, w)$ établies expérimentalement, on a calculé l'ordre de grandeur du travail de mouillage pour les différents sols et roches :

- sable, craie $\ldots \ldots \ldots \ldots \ldots \ldots \ldots \mathscr{f}_{u} \simeq 10^{3} \mathrm{~g} \mathrm{~cm}$

- sable, argileux, silt, loess, kaolin . $\mathcal{L}_{u} \simeq 10^{4} \mathrm{~g} \mathrm{~cm}$

- argiles grasses .............. $\mathscr{E}_{u} \simeq 10^{5} \mathrm{~g} \mathrm{~cm}$ valeurs qui ont été marquées sur l'échelle des énergies (fig. 7). Partant de l'équivalence qui existe entre le travail mécanique et l'énergie calorique (fig. 7), on arrive aux relations entre la valeur intégrale $\left(q_{u}\right)$ et différentielle $\left(q_{u}^{\prime}=\frac{d q_{u}}{d w}\right)$ de mouillage et de travail spécifique de mouillage :

$$
\begin{aligned}
& q_{u}=2.34 \cdot 10^{-5} \mathcal{P}_{u} \\
& q_{u}^{\prime}=2.34 \cdot 10^{-5} \mathrm{~h}
\end{aligned}
$$

et sur cette base, on est arrivé à établir une corrélation (fig. 18) entre les trois méthodes pour caractériser la capacité de rétention de l'eau pour les corps poreux hydrophiles mentionnés plus haut.

En tenant compte des résultats expérimentaux concernant les valeurs de la chaleur différentielle de mouillage $\left(q_{u}^{\prime}=100\right.$ à $\left.600 \mathrm{cal} / \mathrm{g}\right)$ et de la relation $\left(15^{\prime}\right)$, on arrive à la conclusion que la succion correspondante au matériau complètement séché dans l'étuve ne peut être considérée comme égale à $10^{4}$ bars $(p \mathrm{~F} 7)$, pour tous les sols, mais à une variation entre $p \mathrm{~F} 6.63$ et pF 7.40.

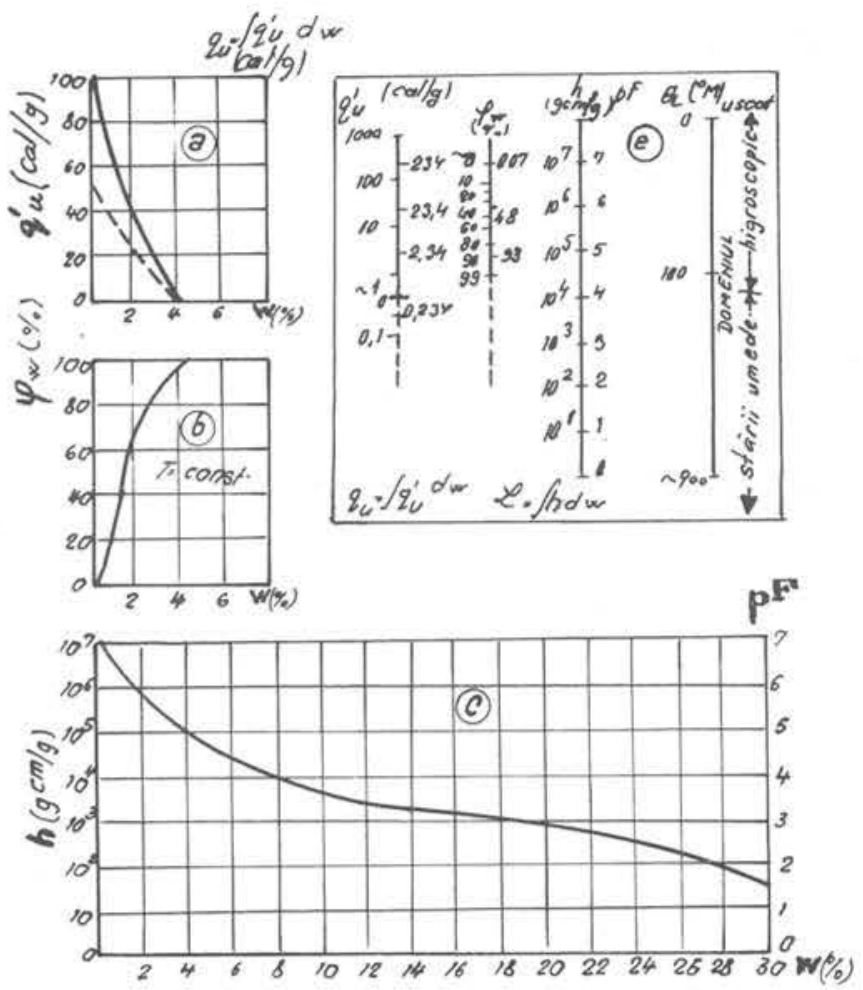

Fig. 18 . 


$$
W_{h}=\theta_{h} \cdot W_{H M}
$$

a).

Fig. 19.

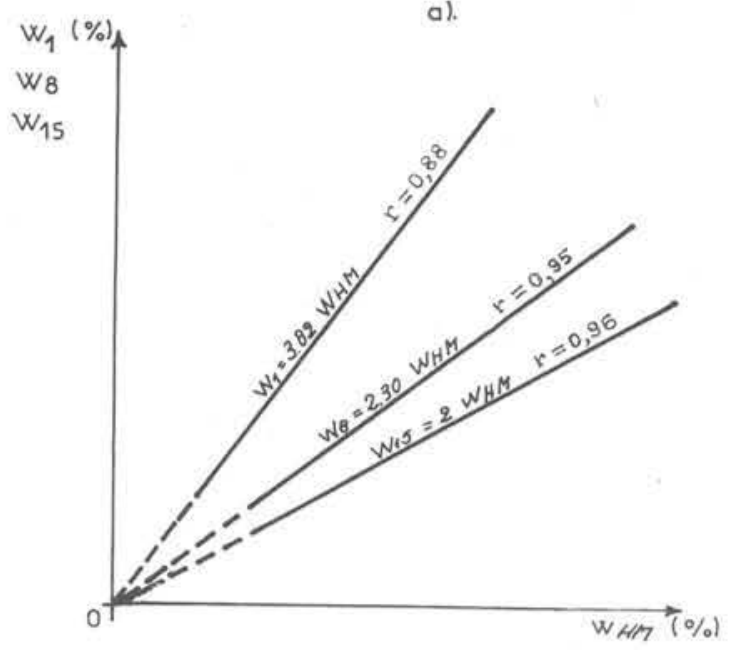

(16)

b).

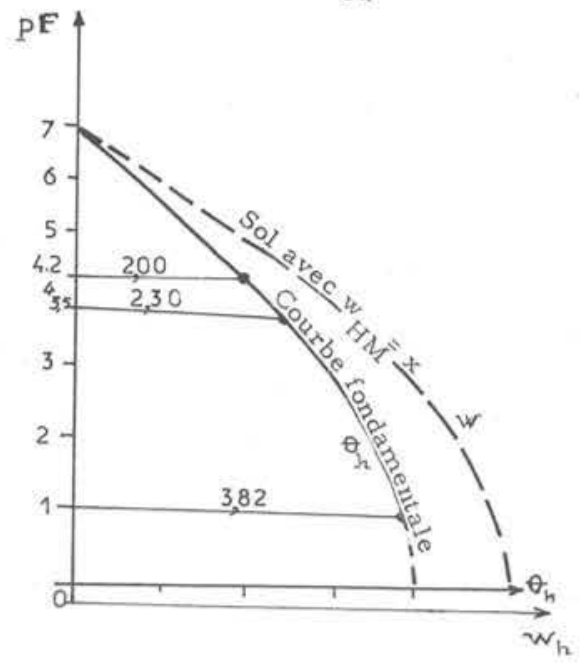

Une autre direction de recherche a concerné les méthodes pour l'établissement de la courbe de rétention. En dehors des méthodes expérimentales, mises au point pour établir la courbe de rétention en imposant certaines succions au sol étudié [4], [7] et pour déterminer la succion momentanée des échantillons [9], on a établi des corrélations linéaires entre la teneur en eau d'hygroscopicité maximale $\left(w_{\mathrm{HM}}\right)$ et les teneurs en eau correspondant à certaines succions ( $h=1.8$ et 15 bars) (fig. 19 a) [12] :

$$
w_{h}=\theta_{h} w_{\mathrm{HM}}
$$

ou $\theta_{h}$ représente un coefficient de proportionnalité qui pratiquement est indépendant de la nature du sol. Il apparaît donc la possibilité d'établir une courbe unique-fondamentale de rétention (fig. 19 b), valable dans le domaine des succions supérieures à 1 bar, où l'influence des modifications de structure (serrage) est moins ressentie; à partir de cette courbe fondamentale $\left(p \mathrm{~F}, \theta_{h}\right)$, valable approximativement pour tous les sols, et connaissant la teneur en eau d'hygroscopicité maximale $\left(w_{\mathrm{HM}}\right)$ du sol considéré, on peut établir tout de suite sa courbe de rétention (fig. $19 \mathrm{~b}$ ).

Le traçage approximatif de la courbe de rétention, sans effectuer des essais de succion et en se basant seulement sur quelques points de la courbe, peut être réalisé en tenant compte de l'existence des corrélations suivantes :

utilise la représentation logarithmique (fig. $20 \mathrm{~b}$ ) et dans lequel en tenant compte de la relation (16), on peut écrire :

$$
\begin{aligned}
p \mathrm{~F}= & \log h=a-b \log w= \\
& a-b\left(\log w_{h}+\log \theta_{h}\right)=c-b \log \theta_{h}
\end{aligned}
$$

où $a, b$ et $c$ sont deś constantes.

On arrive donc, à une expression analytique pour la courbe de rétention (fig. $20 \mathrm{c}$ ) :

$$
h=10^{a} w^{-b}=10^{a} w_{\mathrm{HM}}{ }^{-b} \theta_{h}^{-b}=10^{c} \theta_{h}^{-b}
$$

En admettant que cette relation est vérifiée par les couples des valeurs $p \mathrm{~F} 4.2$ et $\theta_{h}=2.00$ et $p \mathrm{~F} 3$ et $\theta_{h}=3.82$ mentionnés plus haut, on peut établir les valeurs :

$$
c=5.511 \text { et } b=4.92 \text {; }
$$

et l'expression de la courbe fondamentale de rétention devient :

$$
p \mathrm{~F}=5.511-4.92 \log \theta_{h}
$$

qui appliquée pour la succion de 8 bars ( $p$ F 3.92 ; $\theta_{h}=2.30$ ) donne $p \mathrm{~F} \mathrm{3.96}$, donc une précision satisfaisante.

En utilisant les expressions (17') et (17'), on peut donner une expression analytique pour le travail associé à la modification de la teneur en eau :

$$
d \mathcal{E}=10^{a} w^{-b} d w=10^{c} \theta_{h}^{-b} d w=w_{\mathrm{HM}} 10^{c} \theta_{h}^{-b} d \theta_{h}
$$

$$
\begin{aligned}
& h=10^{4} \text { bars } \ldots \ldots, p \mathrm{~F} 7 \ldots \ldots, w=0 \ldots \text { séché à l'étuve ; } \\
& h=30 \text { bars } \ldots . . . \quad p F 4.5 \ldots \ldots w=w_{\mathrm{HM}}-\ldots . \text { hygroscopicité maximale ; } \\
& h=15 \text { bars } \ldots \ldots p \text { F } 4.2 \ldots \ldots, w=2 w_{\mathrm{HM}} \text { flétrissement des plantes ; } \\
& h=8 \text { bars } \ldots \ldots p \text { F } 3.9 \ldots \ldots, w=2.3 w_{\mathrm{HM}} \text {; } \\
& h=1 \text { bar } \ldots \ldots, p \mathrm{~F} 3.0 \ldots \ldots, w=3.82 w_{\mathrm{HM}} \\
& \text { ou } \ldots \ldots \ldots w=w_{p} \text { (en état remanié) ; } \\
& h=1 / 3 \text { bar } \ldots \ldots . p \text { F } 2.5 \ldots \ldots w=4 w_{\mathrm{HM}}+6 \text {; } \\
& h=0.001 \text { bar } \ldots . p F \quad 0.04 \ldots w=w_{\mathrm{L}} \text { (en état remanié) } \\
& \text { ou ...... } w=w_{\text {sat }} \text {. }
\end{aligned}
$$

Les valeur $\theta_{h}=2 ; 2.30$ et 3.82 correspondant aux succions de 15,8 et 1 bars ont été établies sur la base de la corrélation serrée (coefficient de corrélation $r>0.88$ ) constatée pour plusieurs sols de Roumanie.

La détermination de la courbe de rétention à partir de quelques points est facilitée par la linéarisation qui se produit dans le domaine $p \mathrm{~F} 2$ à $p \mathrm{~F} 4.2$ quand on

où :

$$
\mathcal{e}=10^{a} \int w^{-b} d w=w_{\mathrm{HM}} 10^{c} \int \theta_{h}^{-b} d \theta_{h}
$$

donc une proportionnalité entre le travail de mouillage ou drainage et l'avidité du sol pour l'eau, exprimée par l'entremise de l'hygroscopicité maximale $\left(w_{\text {HM }}\right)$. 

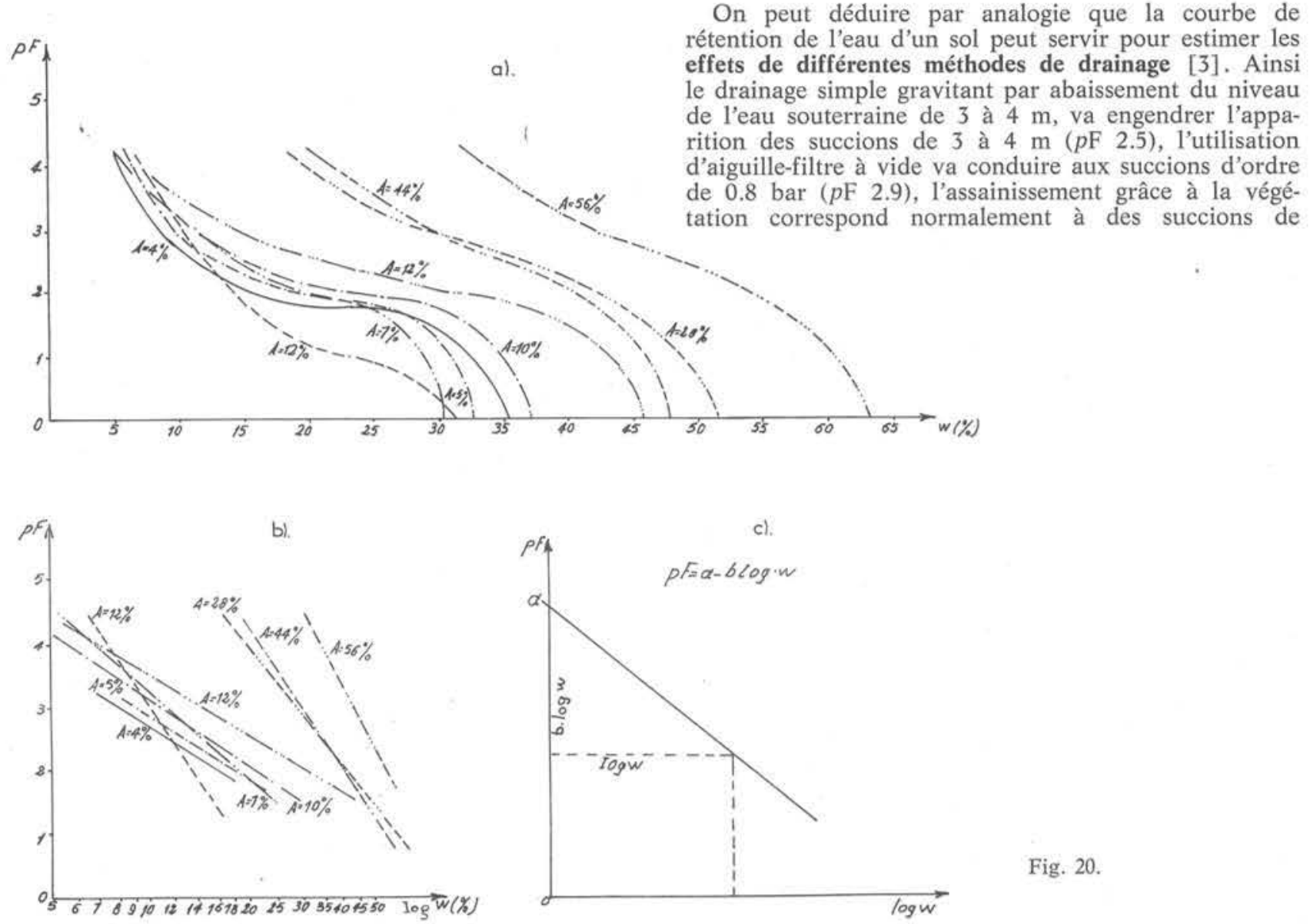

Fig. 20.

Du point de vue historique, la première application de la succion en pédologie a été de caractériser l'accessibilité de l'eau pour les plantes. Ainsi, on a remarqué que le développement des plantes n'est pas lié directement aux valeurs absolues de la teneur en eau du sol mais dépend surtout de l'intensité des forces qui retient l'eau dans le sol, c'est-à-dire de la succion (fig. 21). Par exemple, pour une même teneur en eau de $10 \%$, la succion d'un sol argileux lourd sera plus grande que 15 à 20 bars (le maximum qui peut être développé par les plantes) tandis que pour la même teneur en eau, la succion d'un sol sableux sera beaucoup plus réduite et la plante aura la possibilité d'extraire l'eau nécessaire. Donc, l'accessibilité de l'eau pour les plantes ne dépend pas directement de la teneur en eau du sol mais de sa succion.

Les recherches ont montré également que, à certaines teneurs en eau caractéristiques pour les relations eauplante correspondent pratiquement les mêmes succions. Ainsi, à la capacité du champ $\left(w_{c h}\right)$, c'est-à-dire à la teneur en eau obtenue après l'arrosage suivi d'un drainage du sol pendant quelques jours, correspond généralement une succion de l'ordre de $1 / 3$ bar $(p F 2.5)$; de même le flétrissement permanent d'une plante arrive d'habitude quand la succion du sol dépasse 15 à 20 bars $(p \mathrm{~F} 4,2)$.

Le degré d'accessibilité de l'eau pour les plantes ne reste pas constant dans l'intervalle de l'eau accessible mais diminue d'une manière continue à partir de la capacité de champ vers le point de flétrissement. Le but de l'irrigation est exactement de maintenir les succions réduites en vue d'assumer les conditions optimales pour le développement des plantes.
1-2 bars ( $p \mathrm{~F} 3-p \mathrm{~F} 3.3)$ mais peut s'intensifier pendant les périodes de sécheresse allant jusqu'au 15 bars ( $\mathrm{pF}$ 4.2). Les quantités d'eau éliminées par évapotranspiration des plantes sont appréciables. Ainsi, par exemple, pendant une journée chaude d'été, un chêne puissant peut éliminer jusqu'à 500 litres d'eau. D'ailleurs, c'est un fait bien connu que le défrichage des pentes favorise le déclenchement des glissements.

L'application d'un champ électrique intensifie l'effet d'assèchement. D'après certains exemples de la littérature, un gradient électrique de $0.5 \mathrm{~V} / \mathrm{cm}$ correspond pour un sol silteux à une succion de 3 bars $(p F$ 3.5).

L'utilisation des drains d'aération, prévus avec une circulation d'air, peut théoriquement intensifier le processus d'asséchage jusqu'au domaine hygroscopique pour lequel correspondent des succions supérieures à 30 bars $(p \mathrm{~F} 4.5)$. Ainsi, on peut expliquer pourquoi dans les sols argileux où l'effet des drains simples gravitationnels est presque négligeable (fig. 22), l'adaptation d'un système permettant la circulation d'air peut s'avérer efficace même si on ne peut observer un courant d'eau.

En interprétant les courbes de rétention de quelques dizaines de sols de la vallée du Danube, on a établi le diagramme synthétique représenté sur la figure 23 qui permet de déterminer le degré de saturation $\left(\mathrm{S}_{r}\right)$ obtenu à l'aide de différentes méthodes de drainage pour les sols ayant une plasticité grande $\left(\mathrm{I}_{\mathrm{P}}>35 \%\right)$, moyenne $\left(35 \%>\mathrm{I}_{\mathrm{p}}>20 \%\right)$ et faible $\left(\mathrm{I}_{\mathrm{p}}<10 \%\right)$.

On peut constater que, plus le sol est actif, plus le degré de saturation du sol reste élevé pour une même méthode de drainage. 


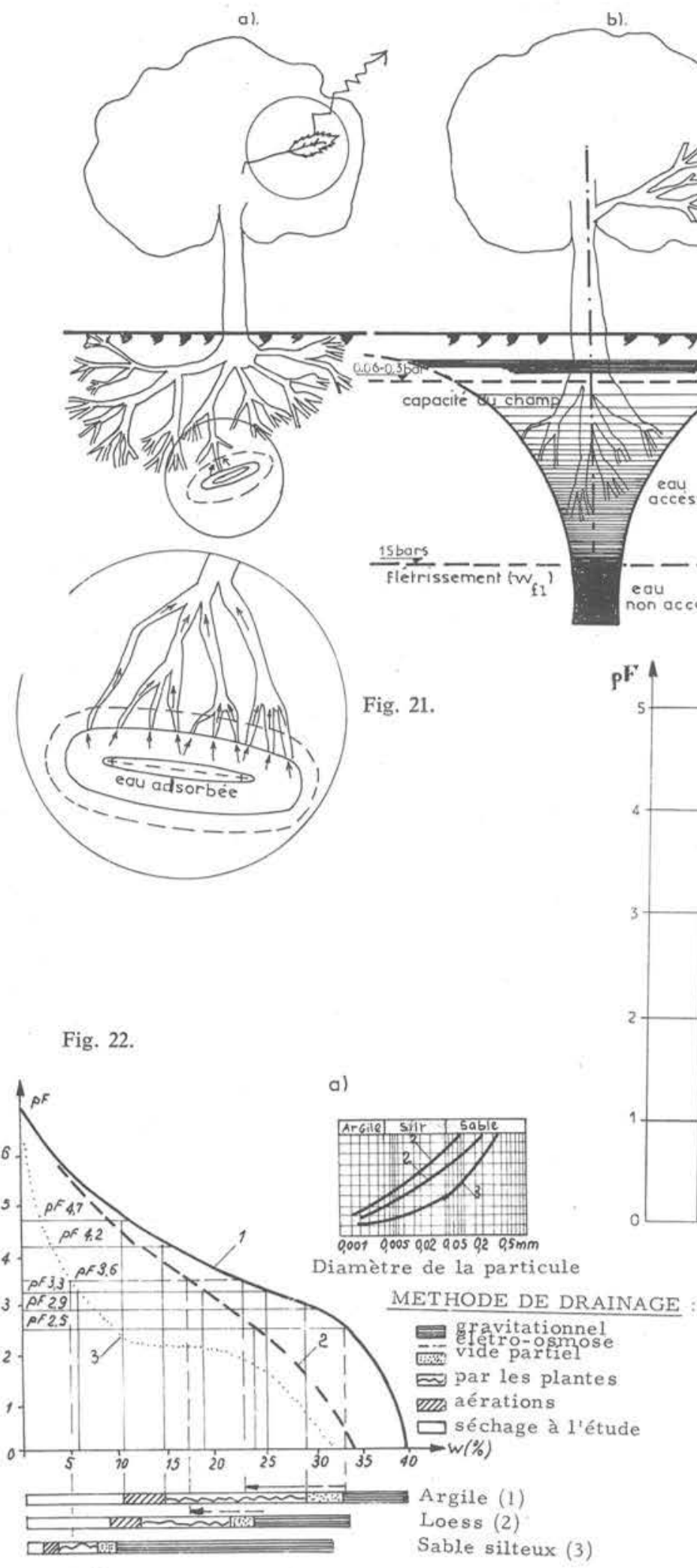

En utilisant la relation (11), on a évalué l'ordre de grandeur du travail spécifique de drainage correspondant aux différentes méthodes appliquées :

- drainage gravitationnel simple ( $p$ F 2.5)

- drainage à l'aide de vide $(p \mathrm{~F} 2.9)$

- drainage par aération ( $p \mathrm{~F} 4.5$ )

$\mathcal{f}_{d r}=10$ à $20 \mathrm{~g} \mathrm{~cm} / \mathrm{g}$

- séchage complet ( $p \mathrm{~F} 7)$ : argiles actives sables fins $f_{d r}^{3}=35$ à $65 \mathrm{~g} \mathrm{~cm} / \mathrm{g}$

$\mathscr{S}_{d r}=10^{2}$ à $10^{3} \mathrm{~g} \mathrm{~cm} / \mathrm{g}$

$L_{d r}=10^{5}$ à $10^{6} \mathrm{~g} \mathrm{~cm} / \mathrm{g}$

$\mathcal{F}_{d r} \simeq 10^{4} \mathrm{~g} \mathrm{~cm} / \mathrm{g}$
Fig. 23. $\quad 10^{5}-10^{6}$ (Argile) $\sim 10^{4}$ (Sable)

b) $\quad h\left(\mathrm{cmH}_{2} \mathrm{O}\right.$

Methode de drainage ${ }^{10^{\mathrm{s}}}$ $10^{2} \div 10^{2}$
On doit remarquer enfin que l'application des moyens de drainage plus intenses est efficace seulement une fois que l'évacuation de l'eau correspondant au drainage simple gravitationnel est assurée.

Le domaine où la succion trouve la plus importante application est celui du mouvement de l'eau dans les sols non-saturés.

Une première question qui peut être résolue en faisant appel à la succion est celle de l'évaluation de la distribution de la teneur en eau d'équilibre sous les revêtements imperméables des routes, aéroports ou sous bâtiments. 
Contrairement à ce qui se passe dans le cas des surfaces libres, lorsque des précipitations de l'évaporation ou de la transpiration des plantes entraîne une migration continue de l'eau entre les différents points du sol, quand il existe un revêtement imperméable (asphalte, feuille de plastique, etc.), le sol va arriver, après un certain temps (dans des conditions isothermes), à une situation d'équilibre; la distribution des teneurs en eau d'équilibre dépend de la position de l'eau souterraine, du régime climatique des propriétés hydrauliques du sol et de l'état d'humidité au moment de la réalisation du revêtement.

Généralement, on distingue les deux cas suivants :

a) quand l'eau souterraine se trouve à une faible profondeur, et quand l'état d'équilibre des teneurs en eau est conditionné par le niveau de l'eau;

b) quand il n'existe pas d'eau souterraine et lorsque l'état d'équilibre dépend principalement des conditions climatiques.

Pour les situations intermédiaires, c'est-à-dire quand l'eau souterraine existe mais qu'elle se trouve à une grande profondeur, la zone d'aération doit être divisée en deux parties : une partie inférieure où l'effet de l'eau souterraine est prédominant et une partie supérieure dans laquelle les conditions climatiques sont essentielles.

Quand l'eau souterraine se trouve à une faible profondeur (jusqu'à $6-9 \mathrm{~m}$ dans les argiles, $3 \mathrm{~m}$ dans les limons et $1 \mathrm{~m}$ dans les sables), la méthode d'évaluation des teneurs en eau d'équilibre mise au point au Road Research Laboratory [13] est basée sur la relation (8). Parmi les facteurs qui interviennent dans cette relation, la pression $p$ qui s'exerce sur l'élément du sol situé à la profondeur $z$ (fig. 22) peut être calculée avec une précision suffisante en utilisant le poids volumique $\gamma$ du sol correspond à une teneur en eau initiale $w_{o}$ :

$$
p=q+\Sigma \gamma z
$$

où $q$ est la charge supplémentaire.

En introduisant la valeur de $p$ dans l'équation (8), il reste trois inconnues $\alpha, h$ et $u$, et si l'on arrive à connaître deux d'entre elles, la troisième résultera. Comme le coefficient $\alpha$ est égal à la pente de la courbe de retrait et la pression interstitielle $u$ est égale à la valeur avec changement de signe de la hauteur du point considéré par rapport au niveau de l'eau souterraine, on déduit tout de suite la valeur $h$ de la succion de l'élément du sol qui, introduite dans la courbe de rétention, nous donne les valeurs $w_{1}$. Ayant les teneurs en eau $w_{1}$ on peut calculer les nouvelles valeurs $\gamma, p$, et après, à l'aide de la courbe de retrait et de rétention, on s'approche par une ou deux itérations des valeurs de teneur en eau d'équilibre.

Pour les sols non-cohérents (sables), le problème devient plus simple, parce que toute la pression est transmise au squelette $(\alpha=0)$, donc la succion sera égale à la hauteur par rapport au niveau de l'eau souterraine. Par conséquent, la distribution d'équilibre de la teneur en eau correspondra exactement à la courbe succionteneur en eau.

La méthode mentionnée plus haut a été vérifiée dans des zones aux climats très différents (précipitations annuelles entre $2400 \mathrm{~mm}$ et $10 \mathrm{~mm}$ ) (22), y compris certaines régions de Roumanie (fig. 24 b).

En utilisant cette méthode nous avons mis en évidence le rôle important de la variation du niveau de l'eau souterraine sur la teneur en eau du fond de la fouille (fig. 25) et par conséquent sur la résistance mécanique du sol.
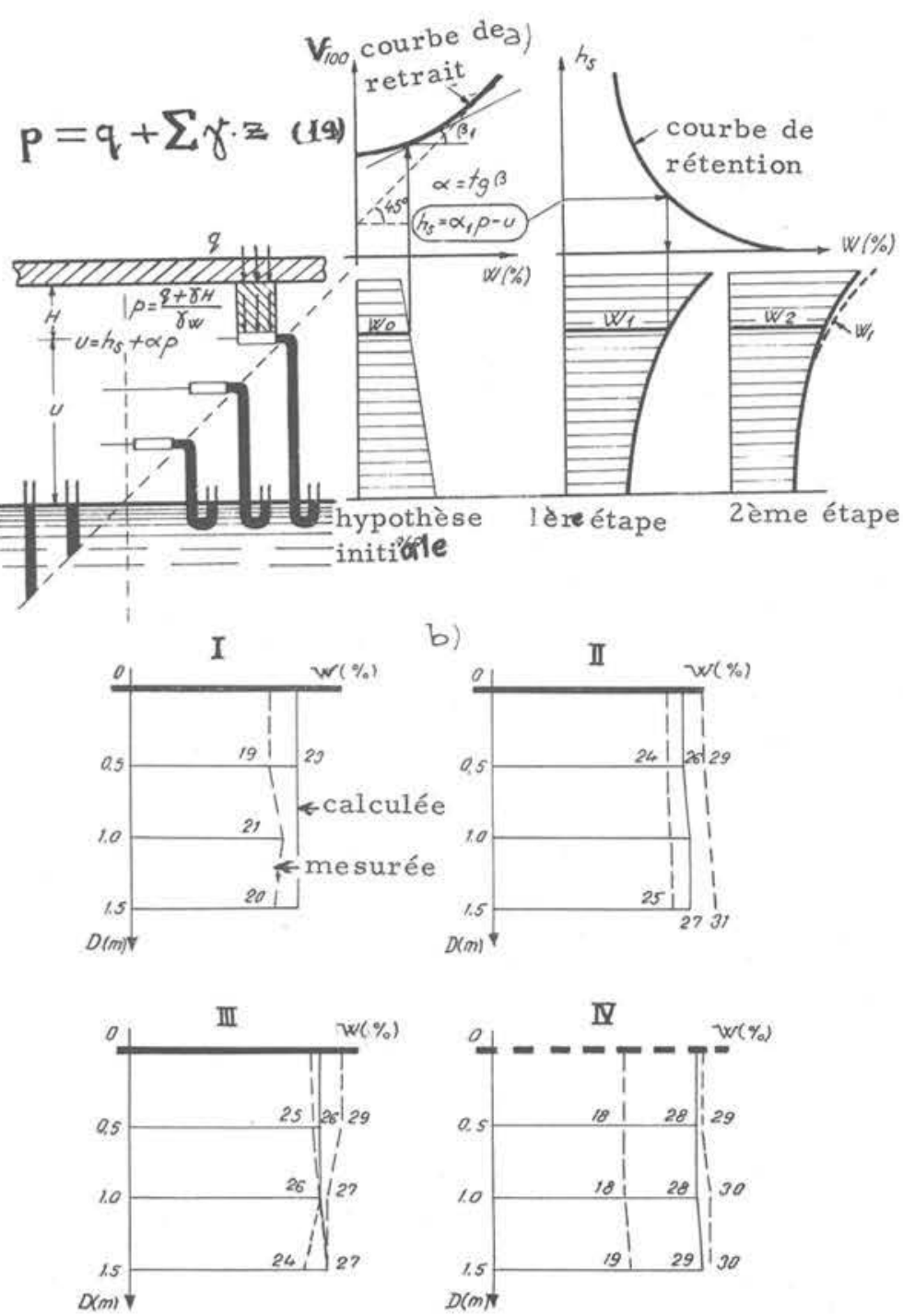

Fig. 24.

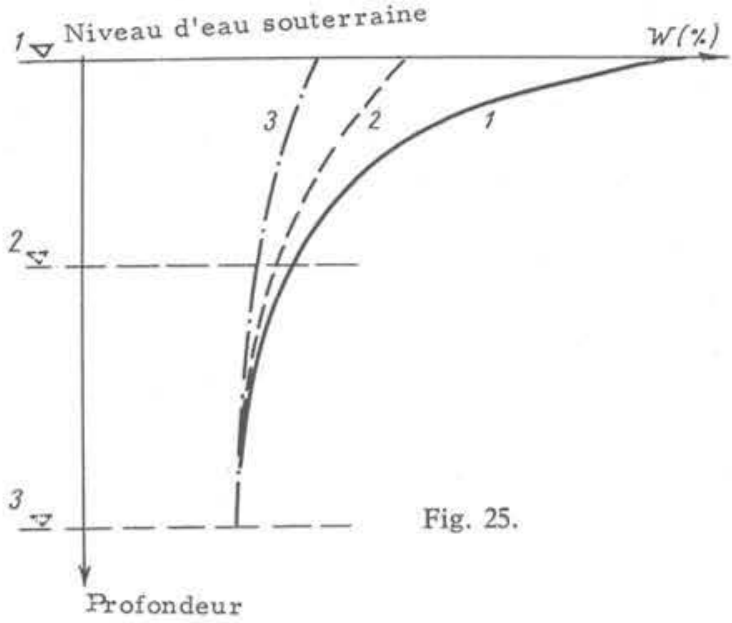

Dans le cas où l'eau souterraine n'existe pas ou bien se trouve à une très grande profondeur, l'état d'équilibre de la teneur en eau dépend des conditions climatiques et spécialement du bilan entre les précipitations (la quantité de l'eau perdue par aire unitaire de la surface du sol à cause de l'évaporation et de la 


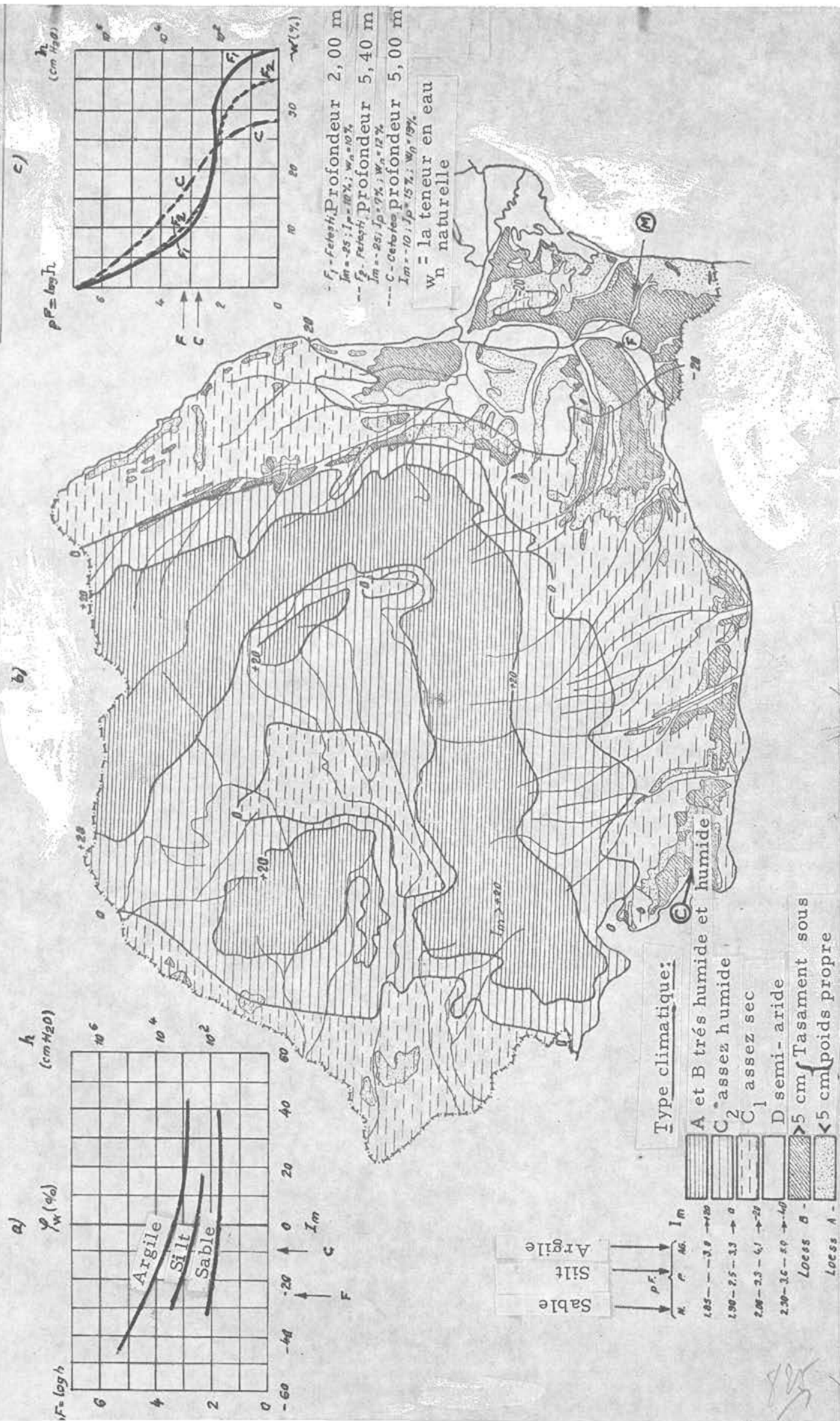

transpiration des plantes). L'état de l'humidité d'équilibre qui se réalisera sous le revêtement imperméable correspondra à des succions déterminées qui dépendent de la nature du sol et de l'indice climatique moyen $\left(\mathrm{I}_{m}\right)$ de Thornthwaite (fig. 26 a) [1]. Cette méthode pour évaluer la distribution des teneurs en eau d'équilibre a été vérifiée avec de bons résultats dans plus de vingt-cinq sites ayant des conditions climatiques très variées $\left(\mathrm{I}_{m}\right.$ de -59 à + 34) [1]. En applijuant cette méthode pour trois sites de Roumanie (C.F.M., fig. 26 b) situés dans des zones de loess, dans lesquelles $I_{m}<0$, on obtient des succions d'équilibre assez élevées ( $p \mathrm{~F} 3.10$ pour Feteshti et $p \mathrm{~F} 2.80$ pour Cetate) qui, introduites dans les courbes de rétention (fig. $26 \mathrm{c})$, conduisent aux valeurs de $10 \%$ et respectivement $20 \%$ qui correspondent assez bien aux teneurs en eau mesurées $(10$ à $12 \%$ pour Feteshti et $19 \%$ pour Cetate).

On remarque sur la figure $26 \mathrm{~b}$, non seulement une bonne coïncidence entre les zones de loess et celles ayant un climat sec $\left(\mathrm{I}_{m}<0\right)$ mais aussi entre la sensibilité du loess au mouillage $\left(i_{m 3}\right)$ et l'aridité du climat. Ainsi, on peut constater qu'aux zones à climat semi-aride $\left(\mathrm{I}_{m}<-20\right)$ correspondent les loess ayant une żrande sensibilité (Dobrogea, Bàràgan, le SudEst de la Moldavie).

Fig. 26. 
Cette constatation est tout à fait explicable si on tient compte que le loess est un sol sous-consolidé, c'est-à-dire à porosité plus grande que celle qui correspond sur la branche principale de la consolidation pour la pression géologique réelle $p_{g}$ (fig. 27 a).

Sa porosité élevée ainsi que sa structure macroporique sont dues généralement aux conditions spéciales qui ont régné pendant sa genèse en climat aride. La poussière de loess, transportée par le vent et constituée principalement par la fraction silt, à laquelle s'ajoutent aussi des grains de sable et des paillettes d'argile, est sédimentée dans des conditions différentes par rapport à celles qui correspondent à la sédimentation dans l'eau. Pour la sédimentation dans l'air, le frottement étant plus grand, la porosité obtenue sera plus élevée. Ultérieurement entre les particules s'établissent des liaisons dues au liant argileux, à la recristallisation du sol dissout dans l'eau interstitielle et aux pellicules de silice qui enveloppent les grains de sable (8). Tant que le loess reste pratiquement $\mathrm{sec}$, sa résistance structurelle reste grande en fonction aussi de la succion élevée de l'eau interstitielle qui mobilise des frottements appréciables.

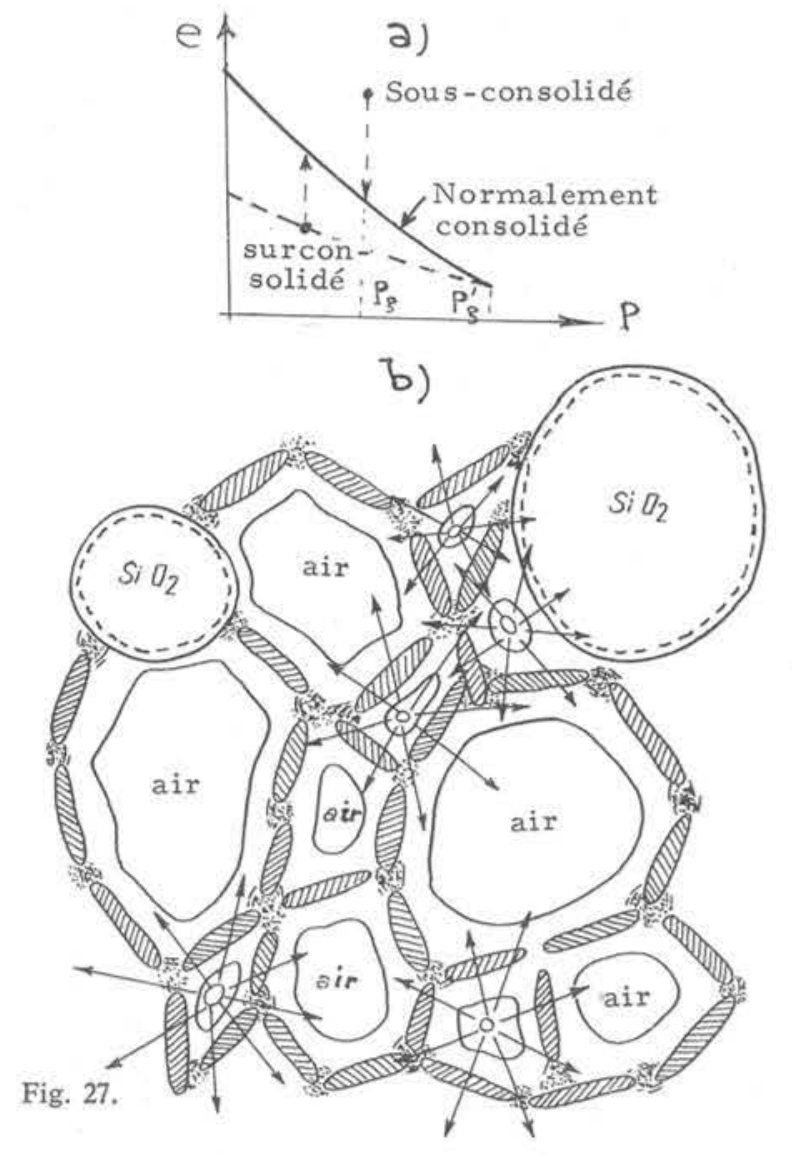

L'inondation du loess diminue ou même anéantit l'effet des facteurs qui contribuent à la résistance de la matrice macroporique; ainsi le liant argileux et le gel de silice vont être mouillés, les sels seront dissouts et la succion va disparaître. La stabilité de la structure ainsi affaiblie est affectée aussi par l'expulsion violente de l'air interstitiel, l'intensité de ce processus variant d'un pore à l'autre (fig. 27 b). Ensuite, la structure du loess va s'écrouler, le matériel ayant tendance à arriver à l'état de consolidation normale.
Le caractère explosif du processus d'humidification des sols secs peut être facilement expliqué si l'on tient compte des énergies qui entrent en jeu pendant le mouillage. Ainsi, si pour $p \mathrm{~F} 3\left(h=1\right.$ bar) $\theta_{1}=3.82$ et pour $p \mathrm{~F} 4.2$ ( $h=15$ bars) $\left(\theta_{2}=2\right.$ en appliquant la relation (12)), on arrive à avoir un travail de mouillage : $\Delta \ell_{1-2}=w_{\mathrm{HM}} \frac{10^{5.511}}{3.92}\left[\frac{1}{2.00^{3.92}}-\frac{1}{3.82^{3.92}}\right]=4560 w_{\mathrm{HM}}$

Comme les recherches ont montré que pour les loess de Roumanie $w_{\mathrm{HM}}=2.65$ à $4.00 \%(0.0265$ à 0.0400$)$ on déduit que :

$$
\Delta \mathrm{e}_{1-2}=121 \text { à } 183 \frac{\mathrm{g} \mathrm{cm}}{\mathrm{g}}
$$

c'est-à-dire une énergie assez élevée dégagée pendant le temps court d'humidification du matériel ayant une perméabilité élevée.

Si la teneur en eau initiale du loess correspond à 1'hygroscopicité maximale $\left(w=w_{\mathrm{HM}}, \theta_{h}=1\right)$ on arrive à des valeurs beaucoup plus élevées :

$$
\Delta C=2180 \text { à } 3200 \mathrm{~g} \mathrm{~cm} / \mathrm{g} \text {. }
$$

En ce qui concerne la distribution des teneurs en eau d'équilibre, on doit mentionner que parfois il est nécessaire d'établir l'état d'équilibre pour deux ou plusieurs matériaux hydrophiles ayant des masses limitées qui sont mises en contact : par exemple le cas de la brique et du mortier qui, ayant des courbes de rétention pour l'eau différentes, arriveront à avoir la même succion finale mais une teneur en eau différente.

Dans ce cas, entre les matériaux hydrophiles mis en contact et qui au commencement étaient aux différentes succions, aưront lieu des échanges de masses d'eau jusqu'au moment où tout le système arrivera à la même succion.

En considérant deux corps poreux hydrophyles A et B (fig. 28 a), isolés par rapport à l'extérieur et qui avaient au commencement des teneurs en eau $w_{o \mathrm{~A}}$ et $w_{o \mathrm{~B}}$, correspondant aux points $\mathrm{A}_{o}$ et $\mathrm{B}_{o}$ sur les courbes de rétention, se pose maintenant la question de trouver les teneurs en eau finales d'équilibre $w_{1 \mathrm{~A}}$ et $w_{1 \mathrm{~B}}$, correspondant aux points $A_{1}$ et $B_{1}$ sur les courbes de rétention pour la situation d'équilibre, quand on arrive à la même succion dans le système constitués par les deux corps. Pour trouver cette succion, on représente sur une figure auxiliaire (fig. 28 b) les courbes $A^{\prime}$ et $B^{\prime}$ qui donnent les masses d'eau $\left(\mathrm{M}_{w}=\mathrm{M}_{s} \frac{100}{w}\right)$ retenues pour différentes succions. Connaissant le point $\mathrm{A}_{o}^{\prime}$, correspondant à l'eau retenue par le corps $\mathrm{A}$ et le point $\mathrm{B}^{\prime}{ }_{o}$, correspondant à l'eau retenue par le corps $\mathrm{B}$, on doit établir maintenant les points $\mathrm{A}_{1}^{\prime}$ et $\mathrm{B}_{1}^{\prime}$ correspondant à la condition que la masse d'eau $\left(\Delta \mathrm{M}_{\mathrm{A}}\right)$ gagnée par le corps A sera égale à la masse d'eau $\left(-\Delta \mathrm{M}_{\mathrm{B}}\right)$ perdue par le corps $\mathrm{B}$. Pour cela, on représente les courbes $\overline{\mathrm{A}^{\prime}}$ et $\overline{\mathrm{B}^{\prime}}$ symétriques de $\mathrm{A}^{\prime}$ et $\mathrm{B}^{\prime}$ par rapport à l'axe mené perpendiculairement sur l'axe des masses dans le point correspondant à la moyenne des deux masses d'eau $M_{A}$ et $M_{B}$.

On remarque facilement que les points $A^{\prime}{ }_{1}$ et $B^{\prime}{ }_{1}$ qui se trouvent à l'intersection des courbes $\mathrm{A}^{\prime}$ et $\overline{\mathrm{B}^{\prime}}$, respectivement $B^{\prime}$ et $\bar{A}^{\prime}$, correspondant aux conditions imposées.

Si l'on a affaire à trois ou plusieurs corps en contact on doit répéter la procédure plusieurs fois (fig. 29).

Un autre domaine de recherche a été celui concernant l'effet des forces de rétention sur les mouvements de l'eau. 


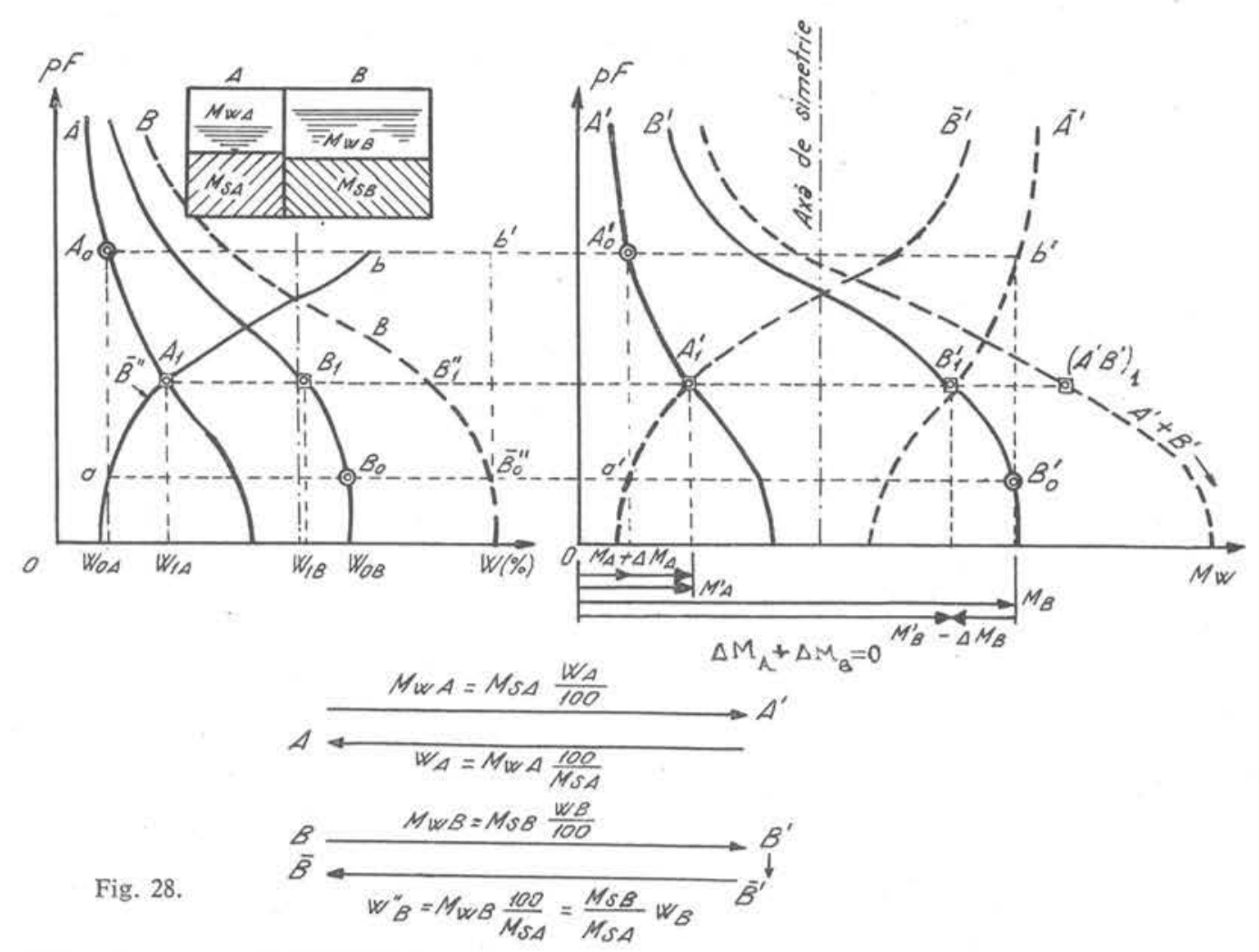

3)

b)

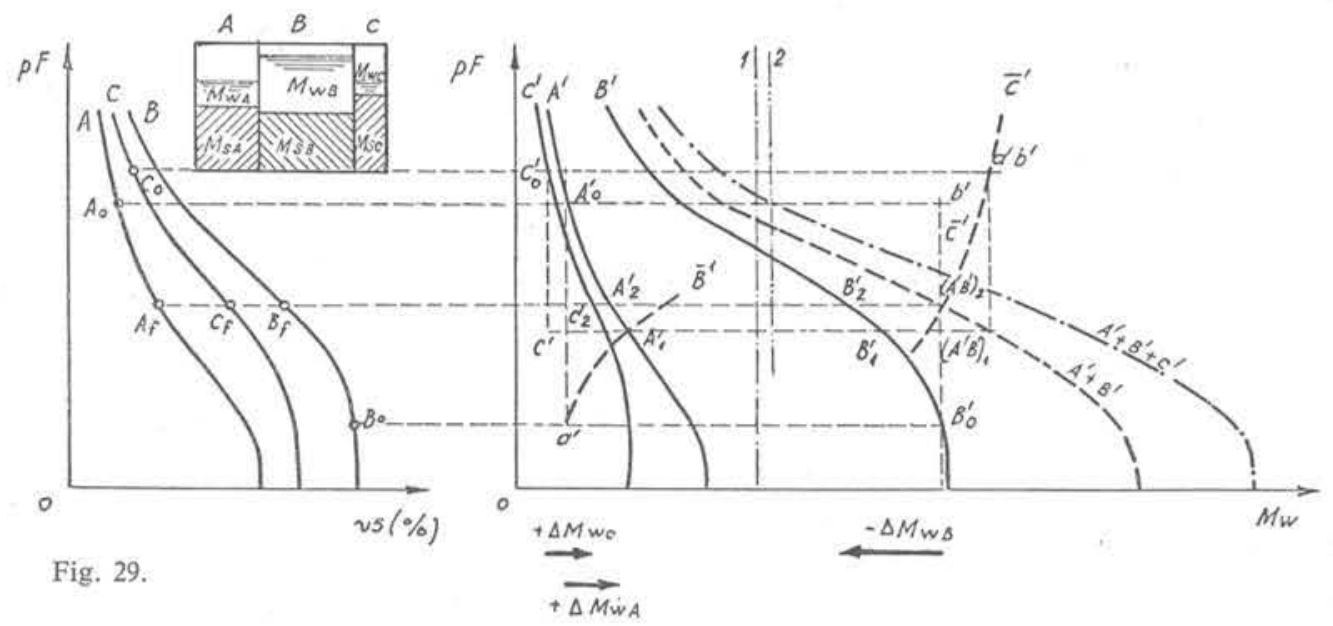

Quand le sol devient non saturé, l'air pénètre dans le sol en diminuant les dimensions des sections occupées par l'eau; en même temps, la diminution de la teneur en eau correspond à un amincissement des pellicules d'eau adsorbée et par conséquent un accroissement des forces avec lesquelles l'eau est retenue par le squelette solide du sol.

Dans cette situation, la perméabilité est fortement influencée par l'état d'humidité du sol et la vitesse de la migration de l'eau est gouvernée par la loi de Darcy généralisée :

où :

$$
\mathrm{V}_{w}=k_{w} i
$$

$k_{w}$, est le coefficient de perméabilité (conductivité hydraulique) ;

$i$, gradient hydraulique dû au potentiel gravitationnel et aux forces de rétention.
Pour les mouvements sur une verticale, la relation (20) devient :

$$
\mathrm{V}_{w}=k_{w} \frac{\Delta h_{g} \pm \Delta h_{s}}{\Delta \mathrm{I}}
$$

$\Delta h_{g}$, différence de potentiel gravitationnel ;

$\Delta h_{s}$, différence de potentiel due aux forces de rétention ;

$\Delta \mathrm{I}$, longueur de la ligne de courant entre les points considérés.

Le signe \pm intervient selon que le potentiel dû aux forces de rétention s'ajoute ou diminue le potentiel gravitationnel.

Par exemple, dans le cas d'infiltration de l'eau dans un massif non-saturé (fig. 30 a) au potentiel gravitationnel s'ajoute la succion de la zone située au-dessous du front d'infiltration. En considérant la teneur en 


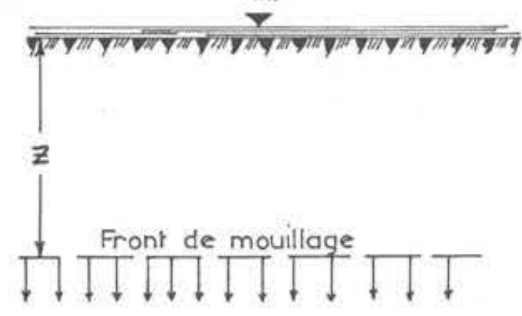

b).
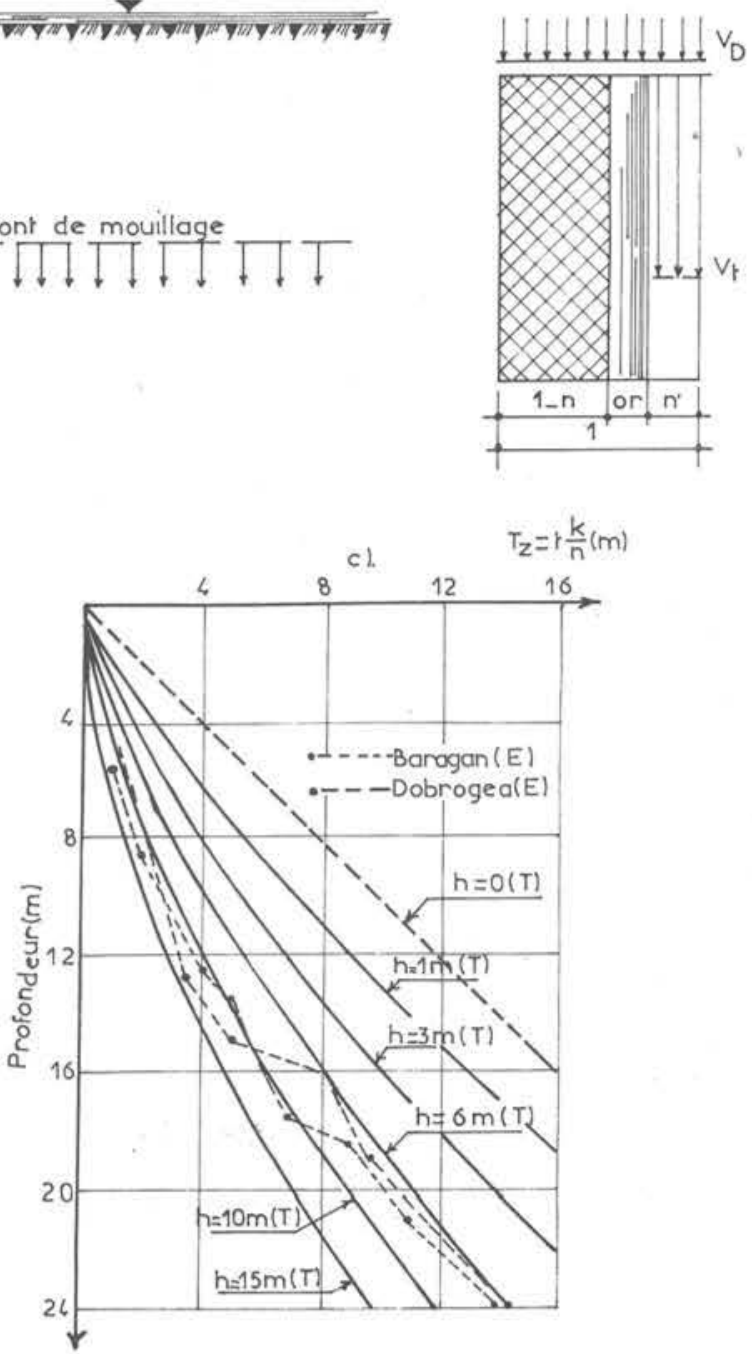

Fig. 30.

eau volumique $(\theta)$ et la porosité libre $n^{\prime}$ (fig. $30 \mathrm{~b}$ ) et en admettant qu'il n'y a pas de variations de volume, on obtient :

où :

$$
\mathrm{V}_{\mathrm{D}} 1=\mathrm{V}_{t} n^{\prime}
$$

$\mathrm{V}_{\mathrm{D}}$ représente la vitesse de Darcy;

$V_{t}$ la vitesse au moment $t$ au niveau du front d'infiltration.

Dans cette condition, la vitesse de propagation de l'humidification à la profondeur $z$ sera :

$$
\mathrm{V}_{t}=\frac{d z}{d t}=\frac{\mathrm{V}_{\mathrm{D}}}{n^{\prime}}=\frac{1}{n^{\prime}} k \frac{z+h}{z}
$$

où

$\mathrm{k}$ est le coefficient de filtration considéré comme constant.

En séparant les variables $t$ et $z$, on obtient :

$$
d t=\frac{n^{\prime}}{k} \frac{z d z}{z+h}
$$

d'où :

$$
\begin{aligned}
t=\frac{n^{\prime}}{k} \int \frac{z d z}{z+h}+\mathrm{C}^{\text {te }}= \\
\frac{n^{\prime}}{k}\left[z-h \operatorname{In}(z+h]+\mathrm{C}^{\text {te }}\right.
\end{aligned}
$$

ou si l'on se met les conditions initiales $(t=o, z=o$ ), on arrive à $\mathrm{C}^{\text {te }}=\frac{n^{\prime} h}{k}$ et l'expression (24) devient :

$$
t=\frac{n^{\prime}}{k}\left[z-h \operatorname{In}\left(1+\frac{z}{h}\right)\right]
$$

et si on fait la notation : $\mathrm{T}_{z}=t \frac{k}{n^{\prime}}$

$$
\mathrm{T}_{z}=z-h \operatorname{In}\left(1+\frac{z}{h}\right)
$$

Cette relation donne la possibilité d'établir le temps nécessaire pour la pénétration du front d'humidification à une certaine profondeur $z$ ou pour l'infiltration d'une certaine quantité d'eau. Pour établir la profondeur $z$ de la zone humidifiée à un certain temps, on peut utiliser un diagramme comme celui montré sur la figure $30 \mathrm{c}$ où sont représentées les courbes d'infiltration pour certaines succions, $h$. L'allure générale de celle-ci résulte de la dérivation de l'expression $\left(25^{\prime}\right)$ par rapport à la profondeur $z$ :

$$
\frac{d \mathrm{~T}}{d z}=1-\frac{1}{1+\frac{z}{h}}=\frac{z}{z+h}
$$

D'après cette expression, on déduit que si la succion est nulle $(h=o)$, la courbe d'infiltration devient une droite inclinée à $45^{\circ}$ par rapport aux axes; plus la succion est grande, plus la courbe d'infiltration est abaissée par rapport à la bissectrice des axes.

En représentant sur ce diagramme les résultats des essais d'infiltration dans les loess de Roumanie (Fetechtien-Baragan et Mircea Vodă en Dobrodgea) [11], on a réussi à déterminer des valeurs de succions équivalentes qui correspondent assez bien aux valeurs qui résultent de courbes de rétention (fig. $26 \mathrm{c}$ ).

$\mathrm{Si}$ on considère par contre le cas d'un massif de sol non-saturé (fig. 31 b) dont la partie inférieure vient en contact avec un niveau d'eau souterraine qui est en train de s'élever, la tendance d'ascension capillaire due à la succion $h$ sera atténuée par l'action retardatrice de la gravitation.

La vitesse d'ascension au moment $t$ sera par conséquent :

$$
\mathrm{V}_{t}=\frac{d z}{d t}=\frac{\mathrm{V}_{\mathrm{D}}}{n^{\prime}}=\frac{1}{n^{\prime}} k \frac{h-z}{z}
$$

où

$k$ est le coefficient de perméabilité considéré comme constant.

En séparant les variables :

$$
d t=\frac{n^{\prime}}{k} \frac{z d z}{h-z}
$$

et en intégrant on obtient :

$t=\frac{n^{\prime}}{k} \int \frac{z d z}{h-z}=\frac{n^{\prime}}{k}[-z-h \operatorname{I} n(h-z)]+c$

Si l'on tient compte de la condition initiale : $t=o, z=o$, on obtient :

$$
\begin{gathered}
c=\frac{n^{\prime}}{k}(h+h \operatorname{In} h) \text { et l'expression (28) devient : } \\
t=\frac{n^{\prime}}{k}\left[h-z+h \operatorname{In} \frac{h}{h-z}\right]
\end{gathered}
$$

où avec la notation : $\mathrm{T}_{2}=t \frac{k}{n^{\prime}}$, on obtient :

$$
\begin{aligned}
\mathrm{T}_{z}=h-z+h \operatorname{In} \frac{h}{h-z}=h \\
{\left[1-\frac{z}{h}-\operatorname{In}\left(1-\frac{z}{h}\right)\right] }
\end{aligned}
$$



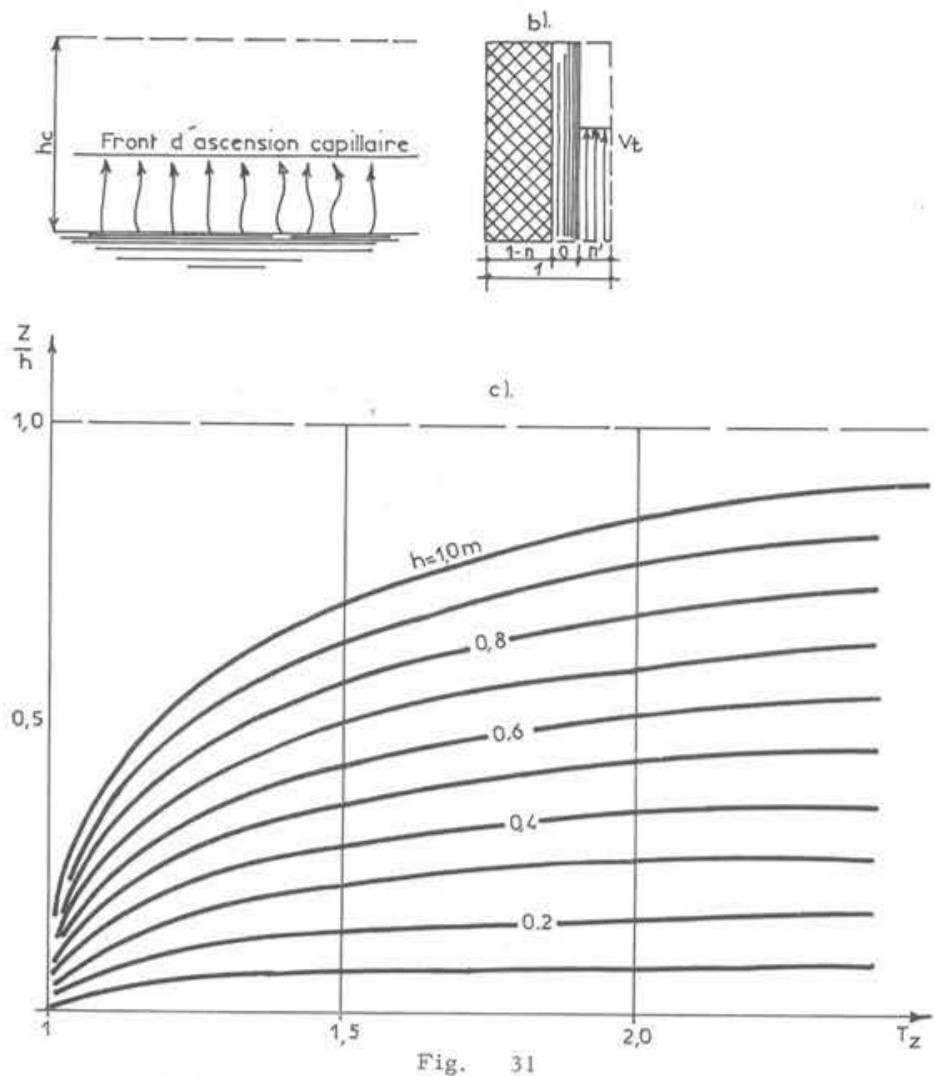

Fig. 31 .

Cette relation donne la possibilité d'analyser la dynamique du processus d'ascension capillaire et de construire des courbes d'ascension capillaires pour différentes succions du massif (fig. $31 \mathrm{~b}$ ). En représentant sur le diagramme quelques points obtenus pendant le commencement du processus, on peut déduire la hauteur finale de l'ascension capillaire $h_{c}$.

Dans le cas du drainage d'un massif par suite d'un abaissement brusque de l'eau souterraine, les forces de rétention ont une tendance opposée à la gravitation et on peut appliquer la loi de Darcy mentionnée plus haut [21] en considérant le signe - au numérateur.

En analysant le cas d'un massif de sable fin, homogène, dans lequel l'eau souterraine qui se trouvait au commencement à la surface, est descendue brusquement de $5 \mathrm{~m}$ et en utilisant la loi de Darcy généralisée on a trouvé que pendant le processus de drainage qui suit on peut distinguer les deux étapes suivantes (fig. 32) [3] :

- la première, qui a une durée relativement courte (cinq jours), pendant laquelle le front de drainage avance assez uniformément et assez vite vers la nouvelle position du niveau de l'eau souterraine, étape pendant laquelle l'eau libre ou retenue par des forces extrêmement faibles (10-15 cm d'eau) est évacuée ;

- la deuxième étape pendant laquelle le rythme de drainage du massif se ralentit continuellement, les courbes de distribution de la teneur en eau à différents intervalles de temps étant en quelque sorte parallèles, avec une légère tendance à augmenter leur pente en vue de la superposition à la courbe de distribution d'équilibre de la teneur en eau dans

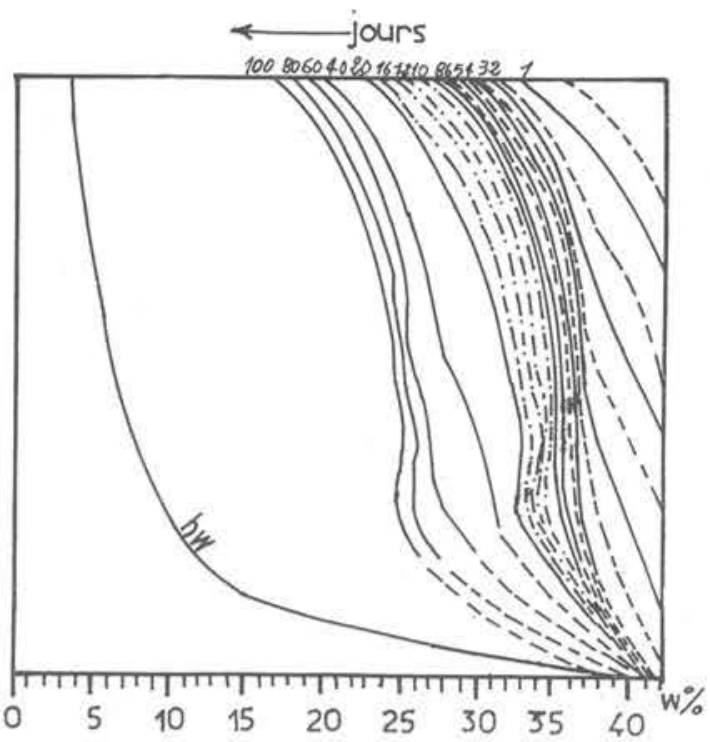

Fig. 32 .

un temps infini, courbe qui dans les cas des matériaux à structure rigide ou pratiquement incompressible $(\alpha=0)$ est justement la courbe de rétention $(h, w)$.

On doit remarquer que la teneur en eau après la première étape est approximativement uniforme et correspond à la capacité du champ $\left(w_{c h}\right)$.

Généralement dans les phénomènes de la migration de l'eau dans les sols non-saturés (fig. 33 a), on peut séparer les deux aspects :

- filtration ou simple transfert de l'eau à travers l'élément du sol considéré (fig. 33 b) ;

- drainage ou mouillage, c'est-à-dire réduction ou augmentation de la teneur en eau (fig. $33 \mathrm{c}$ ), processus liés à l'action des forces d'interaction entre les phases constituant le sol.

a)

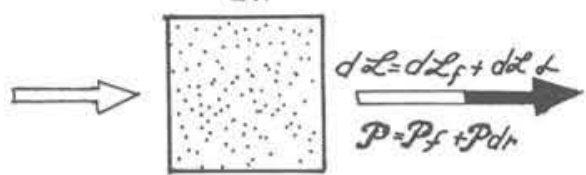

b).

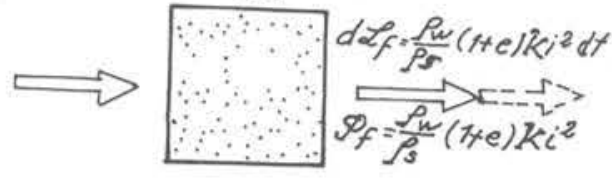

c).

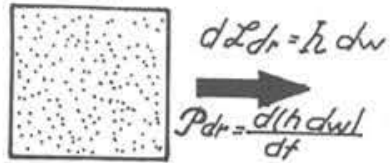

Fig. 33.

On peut démontrer que les échanges d'énergie (correspondant au volume unitaire) liés au processus de filtration seront 


$$
\begin{gathered}
d \mathscr{E}_{f}=\frac{\rho_{w}}{\rho_{s}}(1+e) k i^{2} d t \\
\mathscr{Q}_{f}=\frac{d \mathscr{L}_{S}}{d t}=\frac{\rho_{w}}{P_{s}}(1+e) k i^{2}
\end{gathered}
$$

où

$\rho_{w}$ et $\rho_{s}$, sont les densités de l'eau et du squelette solide :

$e$, l'indice de vide ;

$k$, le coefficient de perméabilité ;

$i$, le gradient hydraulique;

$t$, le temps.

Les notions de travail $\left(\mathscr{E}_{f}\right)$ et de puissance spécifique $\left(\mathscr{S}_{f}\right)$ de filtration, respectivement de drainage ou mouillage (les relations 11 et 13 ) sont utiles pour approfondir les processus de migration de l'eau à travers les sols non saturés, comme c'est, par exemple, le cas d'abaissement du niveau de l'eau souterraine dans les sols à granulation fine.

Contrairement au cas de l'abaissement du niveau de l'eau souterraine dans les graviers, quand l'énergie développée par les pompes est dépensée pour vaincre la résistance à la filtration du sol et la résistance du système de pompage, dans le sol à granulation fine il faut vaincre, en plus, les forces de rétention qui s'opposent au drainage. Il résulte de la relation (11) que cette dépense supplémentaire d'énergie se produit aussi pendant toute la durée de variation de la teneur en eau, c'est-à-dire jusqu'à l'instauration d'un régime permanent d'écoulement. Par exemple, pour l'abaissement de l'eau souterraine dans un sable fin, on a calculé que l'énergie nécessaire au drainage correspond à environ cinq heures de fonctionnement des pompes pour maintenir le niveau abaissé par la filtration. Mais comme en réalité, puisque l'on dépense simultanément l'énergie pour la filtration et pour le drainage, l'effet de retardement des forces de rétention se manifeste pendant une durée beaucoup plus grande.

L'introduction des notions énergétiques permet aussi d'expliquer mieux les processus de migration de l'eau.

La caractéristique de l'accessibilité de l'eau seulement d'après la succion ou l'énergie libre ne donne pas une image complète de la quantité d'eau qui est disponible dans les sols. Ce fait est confirmé par les essais de Peters [21], qui a observé les croissances des racines de maïs en vingt-quatre heures pour différents mélanges des sols et pour différentes succions (fig. 34). On observe que pour les mêmes succions correspondent, en fonction de la texture, différents contenus d'eau et différentes croissances des racines. Donc pour caractériser complètement l'accessibilité de l'eau pour les plantes on doit tenir compte de la succion du sol ainsi que de la quantité d'eau disponible à différents niveaux énergétiques.

Pour dépasser cette difficulté, nous avons proposé la conception dynamique suivante sur l'accessibilité de l'eau pour les plantes.

Comme on le sait déjà, l'alimentation normale des plantes suppose la consommation de quantités bien déterminées d'eau dans certains intervalles de temps. D'où le problème d'assumer un débit nécessaire d'eau qui dépende de plusieurs facteurs, comme l'espèce et l'âge de la plante, le stade de végétation, la température et la teneur en eau du sol et de l'air.

$\mathrm{Si}$ on suppose que la force motrice du processus de migration est représentée par la différence de succion

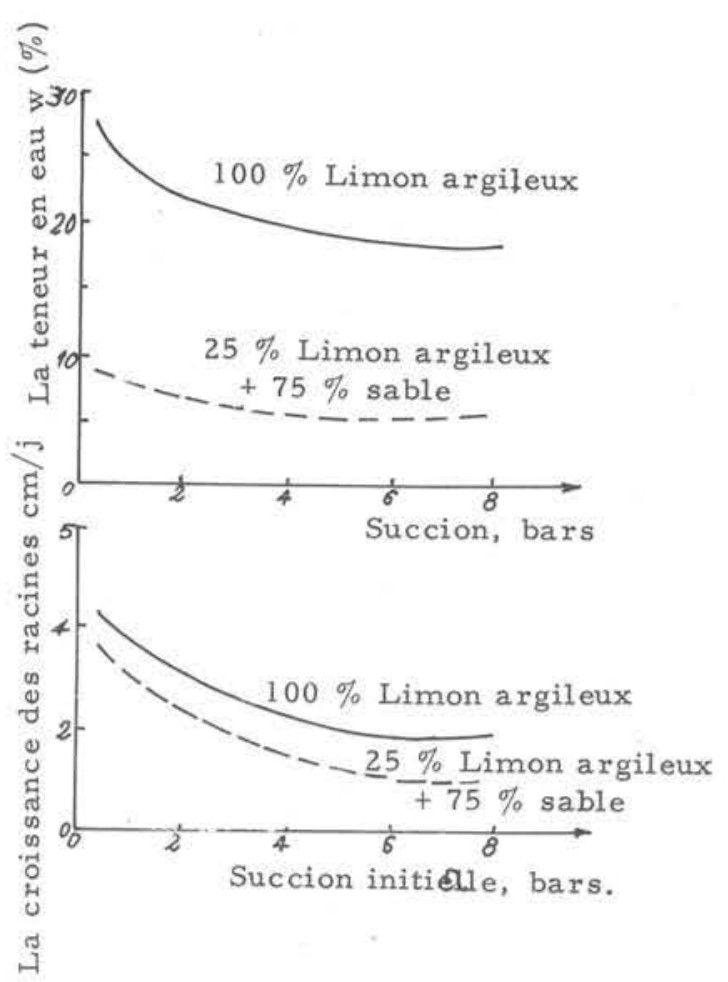

Fig. 34.

$h=h_{r}-h_{s}$ entre la succion des racines et celles du sol on obtient d'après la loi de Darcy généralisée la vitesse de migration de l'eau vers les racines :

$$
\mathrm{V}_{w}=k \frac{\Delta h}{\Delta 1}=k_{w} \frac{h_{r}-h_{s}}{\Delta 1}
$$

où $k$, est la conductivité hydraulique du sol, $\Delta 1$, la distance entre la racine et le point considéré.

$\mathrm{Si}$ on considère $h_{r}$ comme constant, on remarque que le produit des facteurs contenus dans la relation (32) dépend de la valeur de la succion du sol. Quand la teneur en eau du sol est élevée, $k_{w}$ est grand et $h_{s}$ est petit, donc la valeur du produit est assez élevée. Quand la teneur en eau du sol est réduite, $k_{\mathrm{w}}$ diminue et $h_{s}$ croît, et par conséquent, la valeur du produit diminue. Ainsi, si malgré l'effet des racines, la vitesse d'alimentation en eau diminue sous une certaine limite, les besoins en eau ne peuvent être assurés, la plante commence à souffrir et enfin se flétrit.

Donc l'assurance de la vie de la plante dépend de la capacité à assurer un certain écoulement vers ses racines. La puissance développée par la plante est utilisée à vaincre la résistance de drainage $\left(\mathcal{S}_{d r}\right)$ et de filtration $(\mathscr{Q} f)$. On peut donc écrire le bilan :

ou :

$$
\mathscr{Q}_{r}=\mathscr{T}_{d r}+\mathscr{T}_{f}
$$

$$
\mathscr{Q}_{r}=\frac{d \mathscr{L}_{d r}}{d t}+\frac{d \varphi^{\mathcal{C}}}{d t}
$$

ou en tenant compte de (13) et (31)

$$
\mathscr{P}_{r}=\frac{d\left(h d_{w}\right)}{d t}+\frac{P_{w}}{P_{s}}(1+e) k i^{2}
$$

On remarque que cette conception dynamique concernant l'accessibilité de l'eau vers les racines des plantes présente l'avantage de réunir l'aspect qualitatif (succion) et l'aspect quantitatif (teneur en eau). 


\section{PROPRIETES MECANIQUES DES SOLS NON SATURES}

L'élément essentiel qui conditionne les propriétés mécaniques des sols non saturés est la succion de l'eau interstitielle. Ce fait est par exemple bien illustré par les résultats expérimentaux concernant la variation de la résistance au cisaillement établie à l'aide du scissomètre en fonction de la teneur en eau (fig. 35 a) et de la succion (fig. 35 b) [14]; on peut constater pour les mélanges de sable et de kaolinite $(C)$ ou montmorillonite $(M)$ dont les courbes de rétention sont données sur la figure 16 a que pour une même succion (fig. $35 \mathrm{~b}$ ), indépendamment de la nature du minéral argileux, on obtient presque la même résistance.

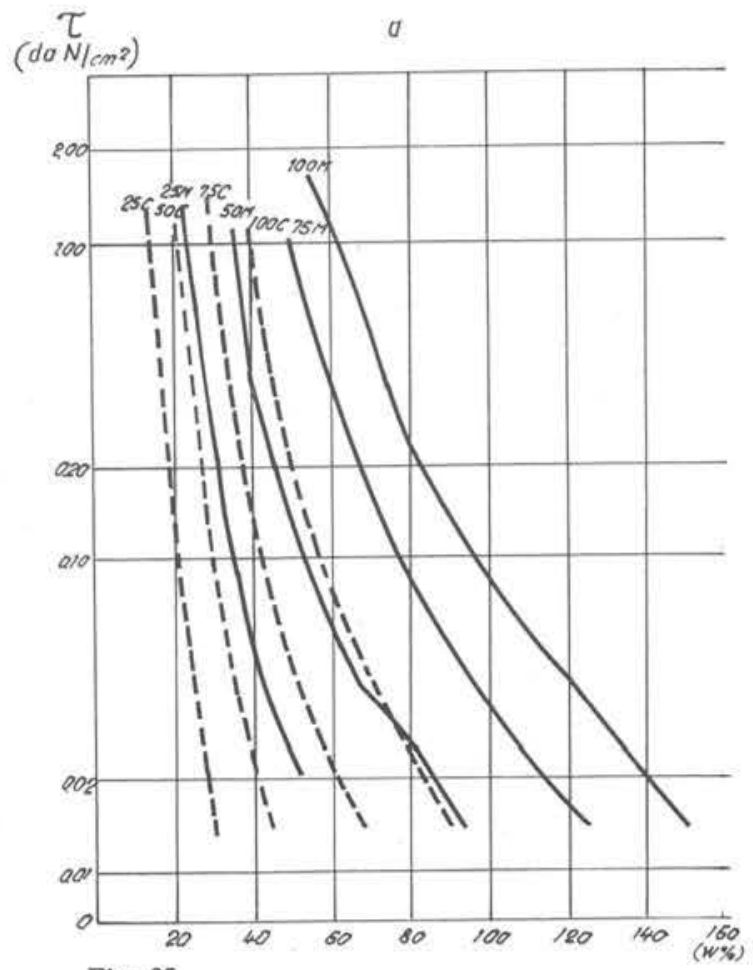

Fig. 35 .

D'ailleurs, ce fait est parfaitement explicable si l'on tient compte du principe de la contrainte effective $\left(p^{\prime}\right)$ qui appliquée aux sols non saturés devient en considérant la relation $(8)$ :

$$
p^{\prime}=p-u=p+h-\alpha \quad p=(1-\alpha) p+h
$$

Donc la contrainte effective a deux composantes : l'une due à la pression extérieure $(p)$ et l'autre à la succion. Au fur et à mesure que l'on s'éloigne de l'état de saturation, $h$ croît rapidement et la contrainte effective qui mobilise la résistance au cisaillement est dominée par la succion.

Comme la succion est influencée en même temps par la teneur en eau et la densité (fig. $16 \mathrm{~b}$ ) pour étudier la variation des propriétés mécaniques des sols non saturés, on a fait appel à l'abaque décrit plus haut (fig. 3). On peut même imaginer un système de référence qui a comme plan de base l'abaque Terracina et sur une normale à ce plan l'indice de la propriété mécanique qui nous intéresse. En admettant que pour chaque état correspond une valeur bien déterminée de l'indice, on arrive à tracer une surface de variation de cet indice. Les intersections de cette surface avec les plans horizontaux d'égal indice vont donner en les projetant sur le plan de base des courbes d'égal indice.
Dans le cas représenté dans la figure 36 , on a affaire à des courbes d'égales résistances à la compression simple ( $\sigma_{c}$ en bars) d'une argile.

L'utilisation de cette abaque nous donne une image plus claire du rôle de l'humidité ou du serrage sur les propriétés des sols non saturés. En ce qui concerne les sols saturés, les modifications de l'indice seront comprises dans le plan perpendiculaire au plan de base ; la trace de ce plan perpendiculaire est la droite de saturation.

Dans ce qui suit, on donne plusieurs exemples pour montrer la manière dont on peut utiliser l'abaque et les conclusions qu'on peut en tirer; pour l'élaboration de ces exemples on a utilisé les résultats de nos recherches [5], [6] ainsi que des données trouvées dans la littérature et qui représentent une source importante pour mieux comprendre le comportement des sols non saturés.

Ainsi, d'après la figure $36 \mathrm{~b}$, où est représentée la variation de la résistance à la compression simple d'une

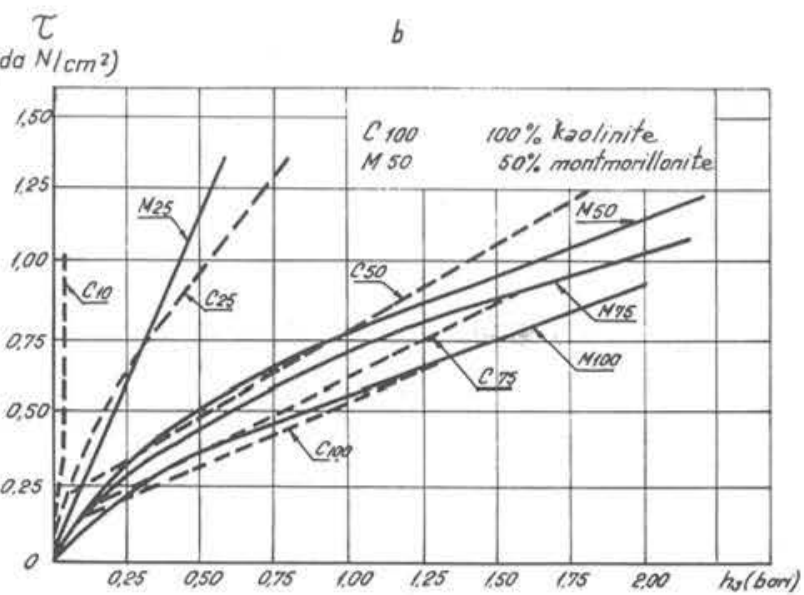

argile, on peut déduire que des modifications de l'état d'humidité et de l'état de serrage ont des influences presque égales.

D'après la figure 37 où sont représentés les résultats des essais sur un sable fin de Părtarlage (Roumanie), on peut déduire que l'influence de la teneur en eau et de la densité sur les résistances à la traction $\left(\sigma_{t}\right)$, au cisaillement $(\tau)$ et sur la cohésion apparente $\left(c_{a}\right)$ a un caractère tout à fait analogue.

En représentant dans l'abaque les données publiées par B.D. Kazarnovaski [16] concernant les paramètres de la résistance au cisaillement $(\Phi$ et $c$ ) d'un silt sableux (fig. $38 \mathrm{a}$ ) et d'une argile silteuse (fig. $38 \mathrm{~b}$ ), on arrive à la conclusion que les valeurs de $\Phi$ sont presque indépendantes de la densité et ressentent vivement l'influence de la teneur en eau. En même temps, on doit remarquer que sur la cohésion $c$ l'influence de l'état d'humidité et de l'état de serrage dépend du domaine considéré. Il est intéressant de mentionner que l'allure des courbes d'égale valeur $\Phi$ et $c$, obtenues en utilisant les données établies par D. Milanovic [19] pour les loess du bassin de Danube, conduit à des conclusions analogues. 


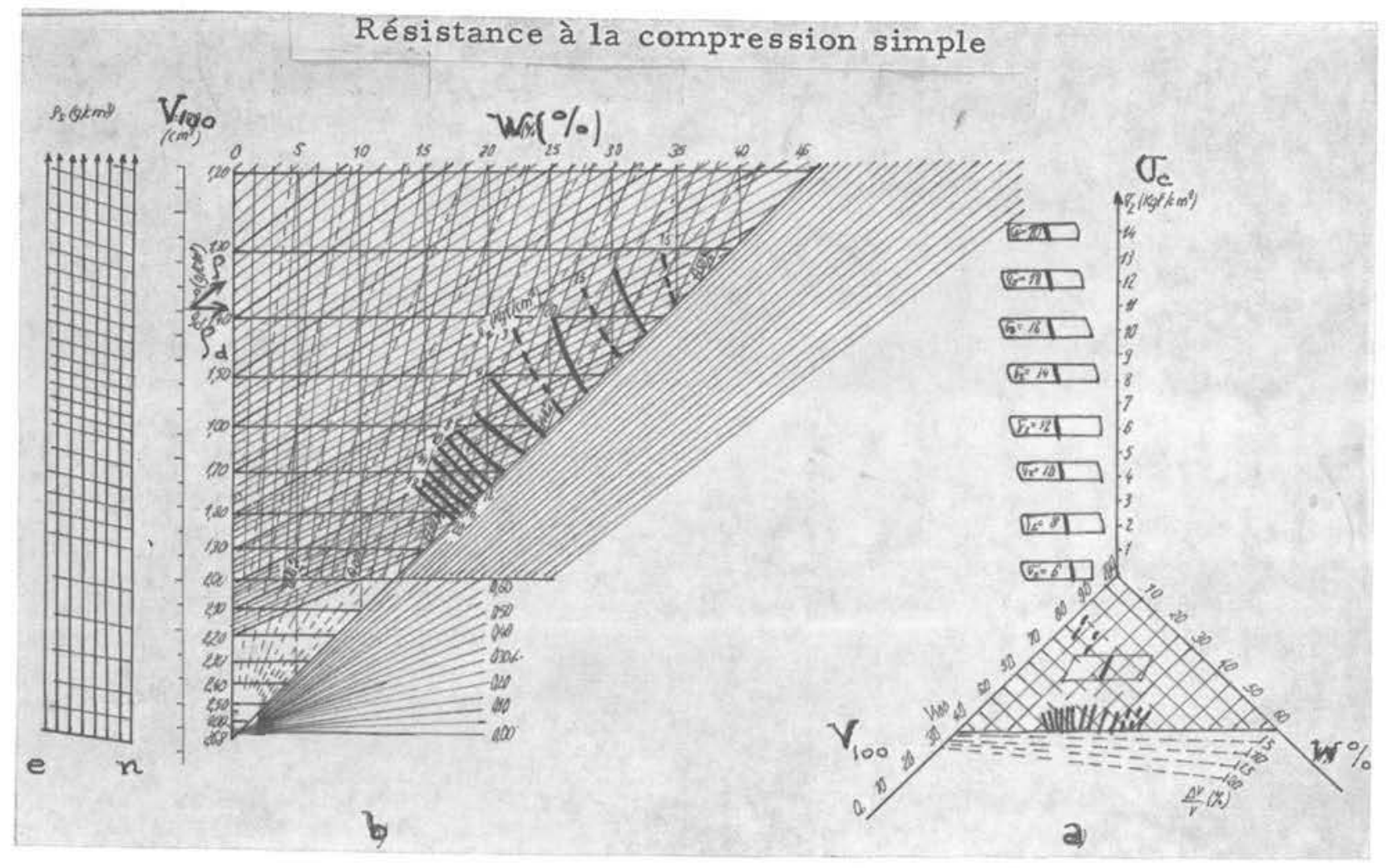

Fig. 36.
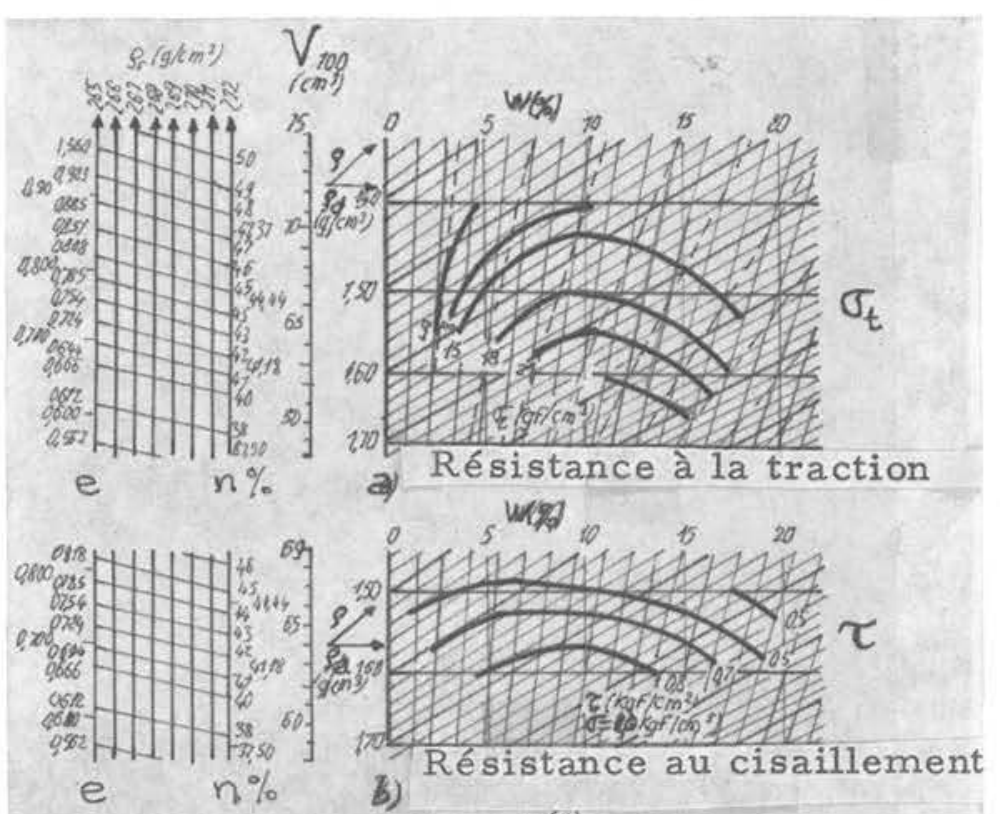

Fig. 37.

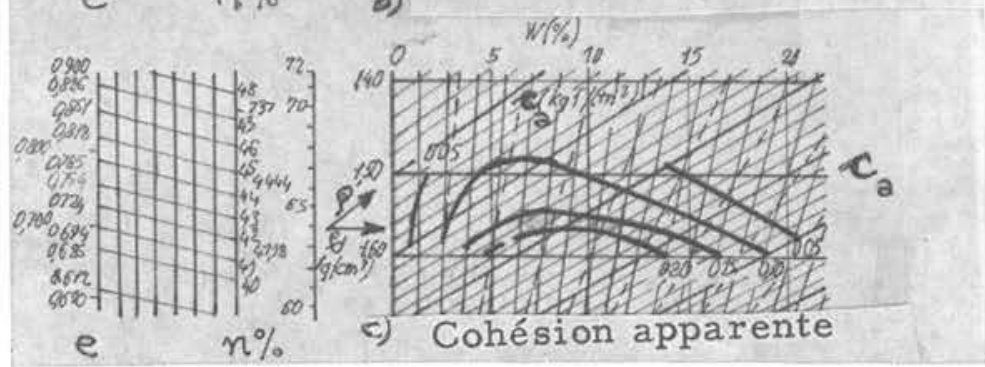

D'après la figure 40 où sont représentées les courbes d'égales modules de la déformation linéaire $\mathrm{E}$ d'un sable argileux compacté [23], on déduit que l'influence de la teneur en eau est beaucoup plus grande que celle de l'état de serrage. Le fait que les courbes E sont presques parallèles conduit à la conclusion, que dans la représentation spaciale mentionnée plus haut, la surface E est presque plane. 


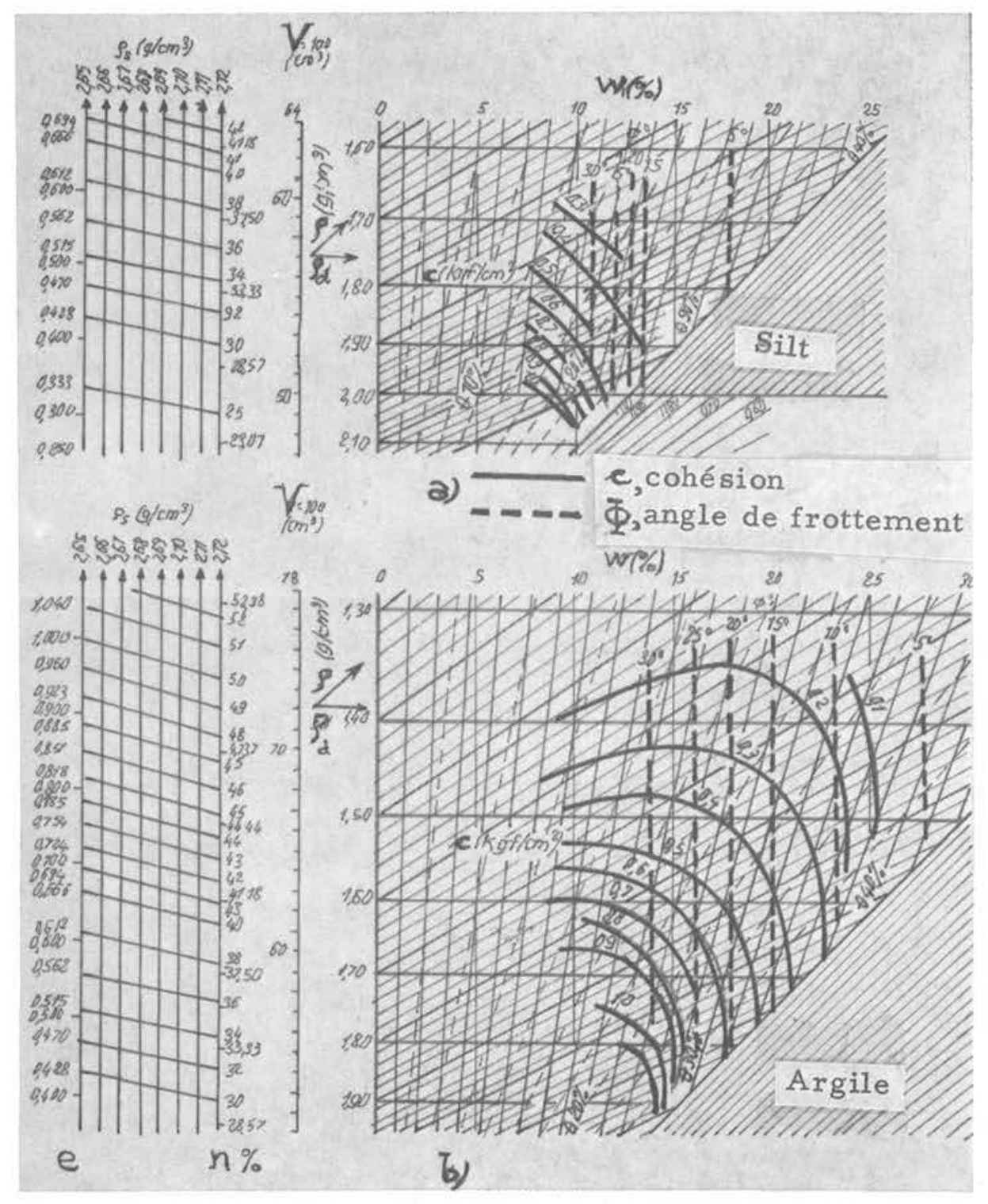

Fig. 38.

Fig. 39.

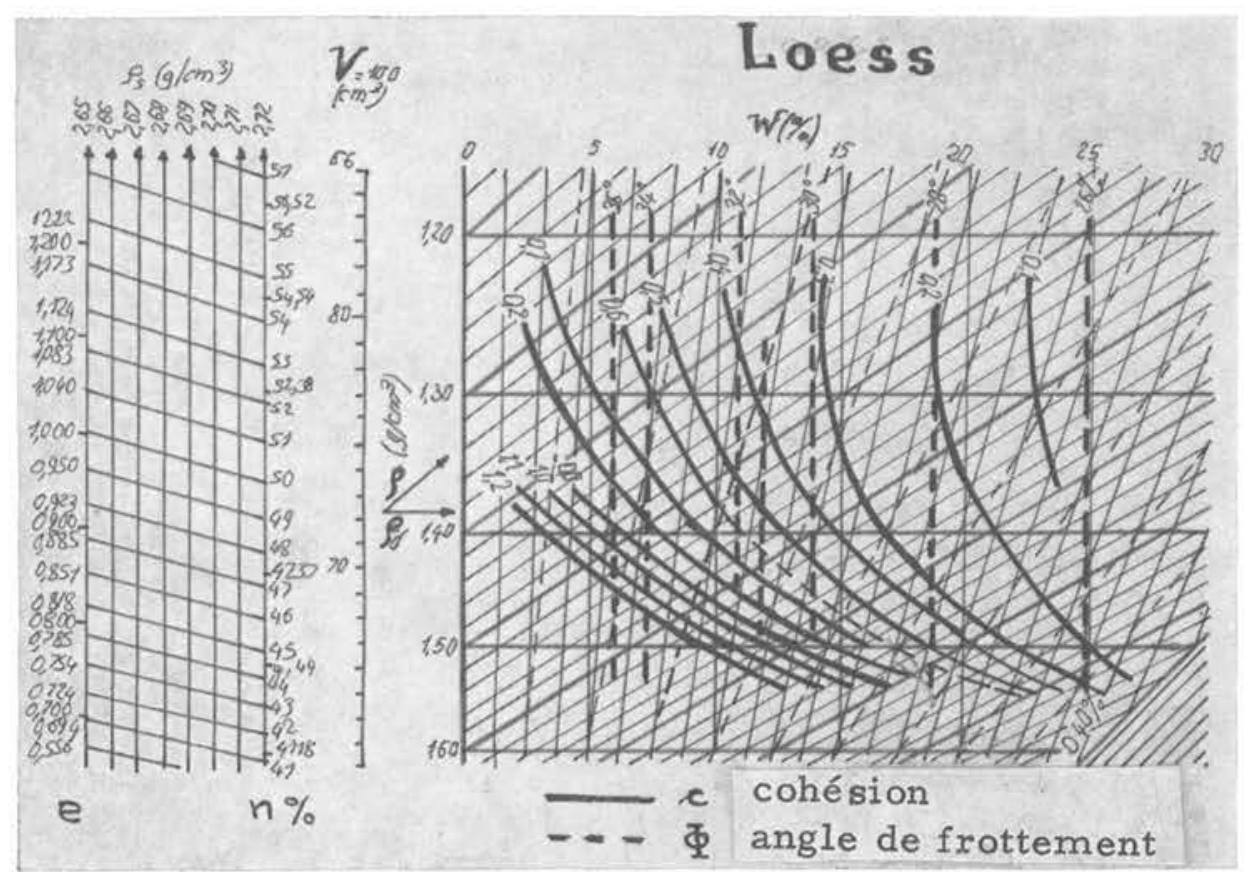



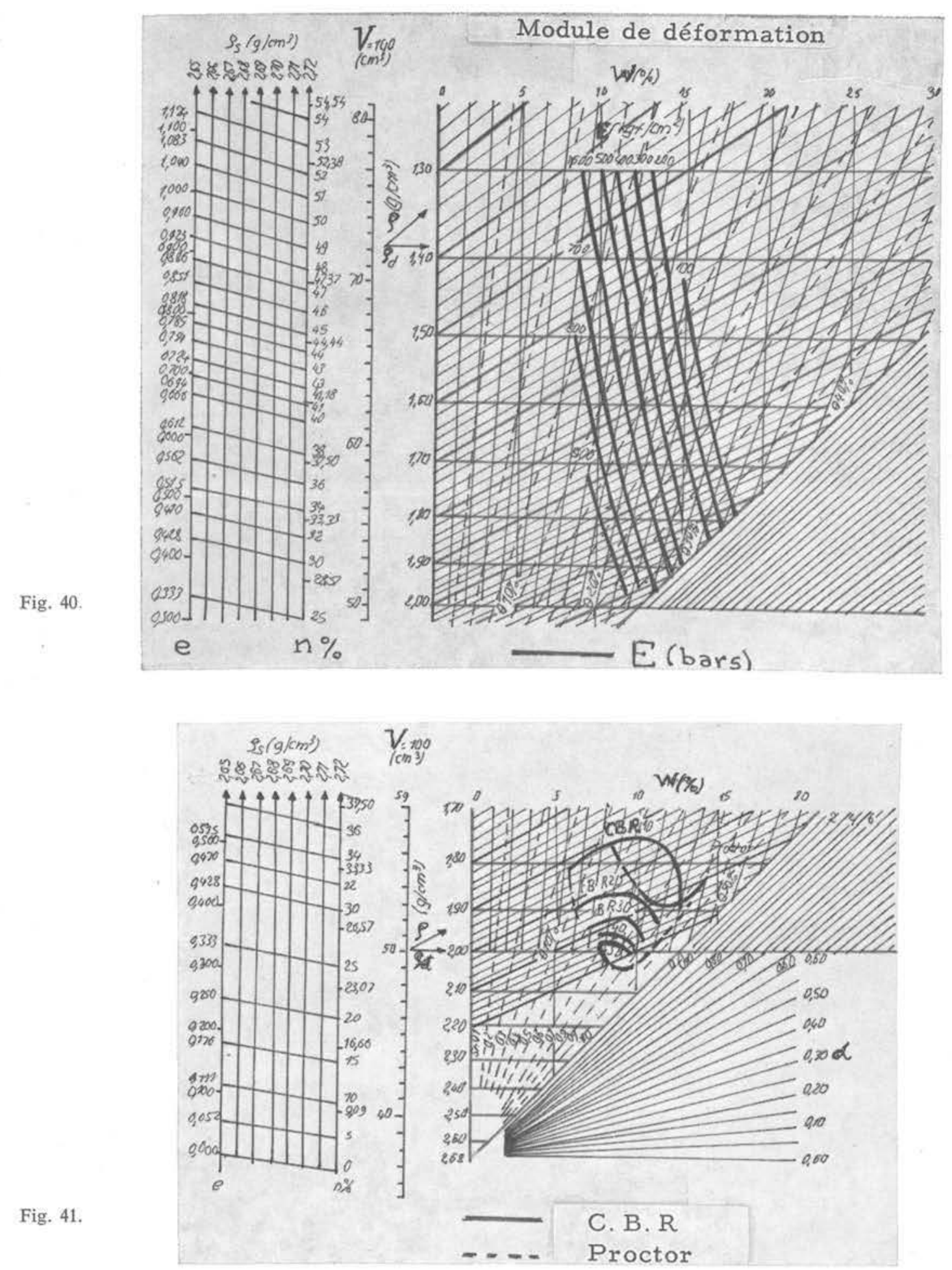

En analysant les deux abaques de la figure 41 , où sont représentées les courbes d'égal indice portant californien $\mathrm{CBR}$, on arrive à la conclusion que l'allure de cette courbe dépend en bonne mesure du type du sol et des autres facteurs qui interviennent pour conditionner un phénomène aussi complexe que la capacité portante d'un sol.

Aussi, en ce qui concerne les argiles actives, on doit signaler que l'abaque nous permet de suivre les variations saisonnières de l'état d'humidité et de serrage.
Ainsi, la figure 42 montre que les modifications de l'état pour une argile active dans un climat aride sont importantes et que le sol devient saturé seulement pendant l'hiver; l'abaque nous montre aussi que le "chemin " parcouru au cours des saisons est assez éloigné de celui obtenu pendant les essais de retrait au laboratoire. Dans ce cas, le sol s'éloigne assez vite de la saturation et les changements entre l'été et l'automne se développent dans la bande comprise entre les droites $\mathrm{S}_{r}=0.7$ et $\mathrm{S}_{r}=0.6$. Ce comportement un peu 


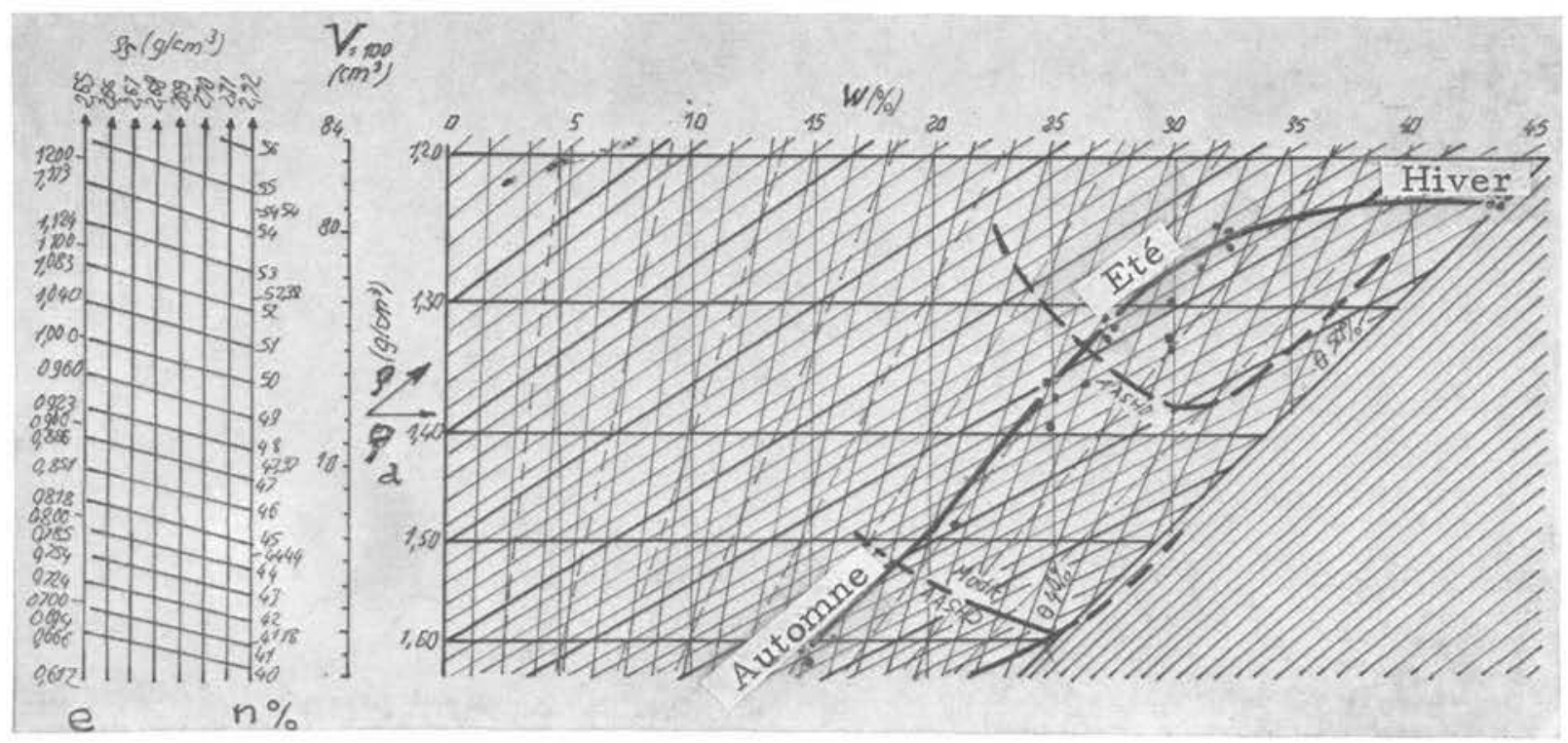

Fig. 42.

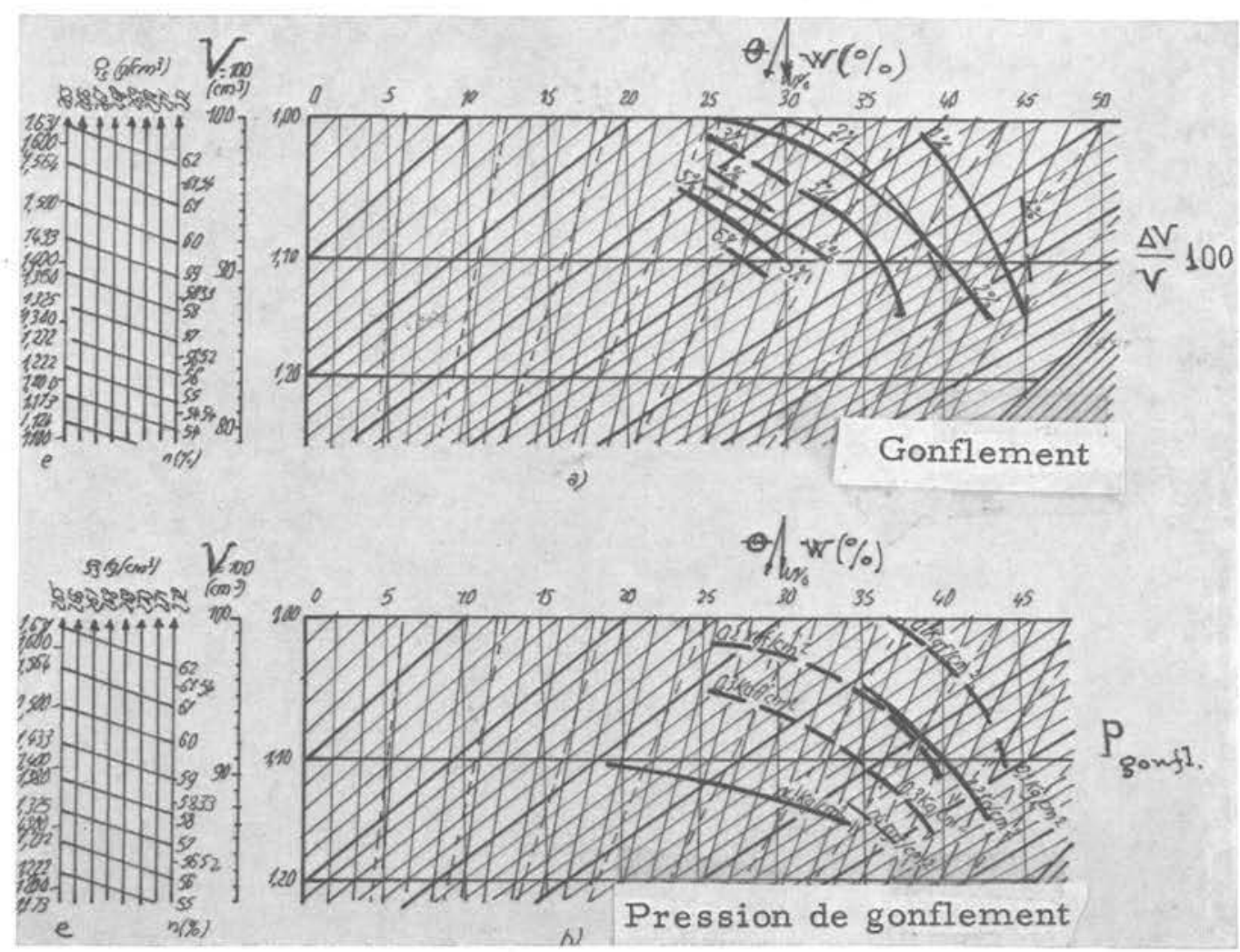

Fig. 43 .

inhabituel reste encore à étudier, mais parmi les causes possibles recherchées, on doit faire intervenir la fissuration et la structuration qu'on observe dans les argiles actives.

La figure 43 montre que pour des échantillons d'argiles actives remaniées [15] les gonflements en pourcentages $(a)$ et les pressions de gonflements $(b)$ restent presque les mêmes indépendamment de la préparation des échantillons (par la modification de la densité ou de la teneur en eau) et dépendent seulement de l'état d'humidité et de serrage au commencement de l'essai.
Enfin, une dernière question sur laquelle il est intéressant de s'arrêter est celle de l'utilisation de l'abaque pour suivre les changements d'état qui interviennent pendant les essais mécaniques. Ainsi, si on représente les résultats obtenus pendant un essai double œdométrique et si sur " les chemins de changement d'état " sont marqués les points correspondant aux différentes pressions appliquées, on peut tracer les courbes d'égale résistance de la structure (fig. 44).

Ces courbes indiquent les teneurs en eau et les densités atteintes à l'état d'équilibre quand le sol est 
Fig. 44.

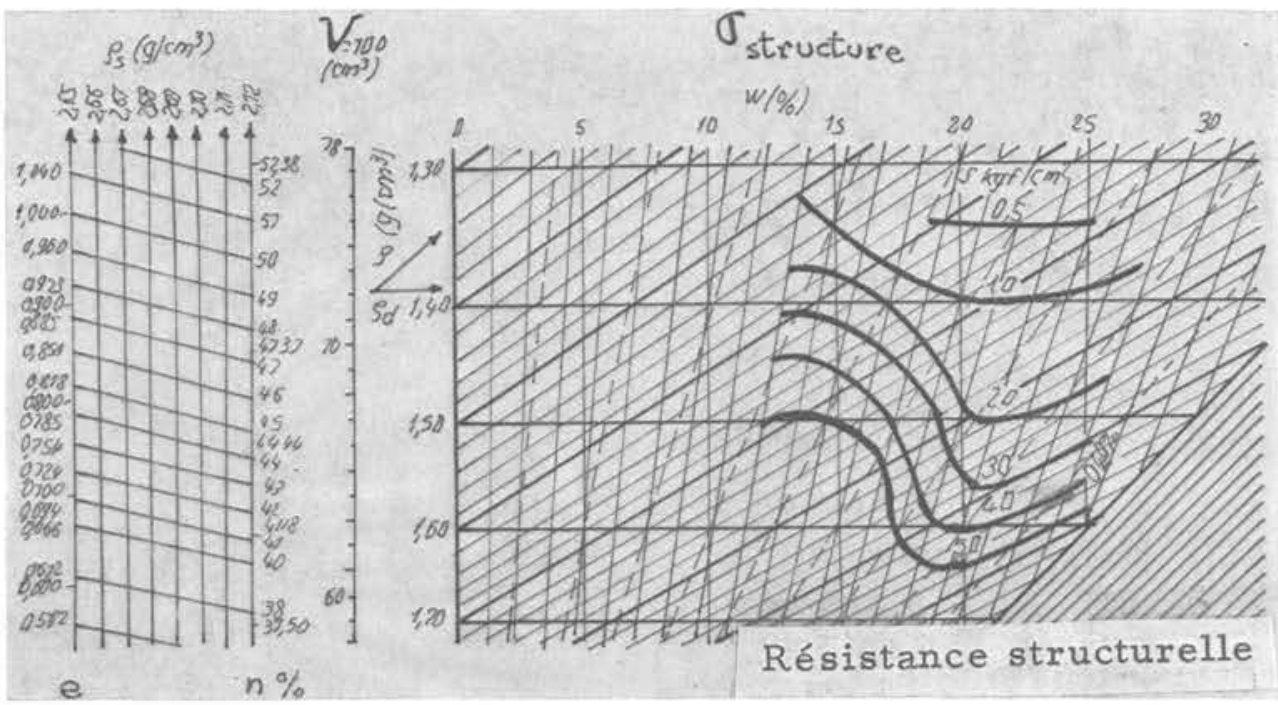

soumis à différentes pressions $p$; la connaissance de ces courbes permet une prévision des modifications de volume engendrées par les changements de l'état. Ainsi, par exemple, si le point $p$, correspondant à l'état du sol qui est soumis à une pression $p$, se trouve au-dessus de la courbe de résistance structurelle, $p$, le sol est insuffisamment consolidé et on doit attendre des tassements supplémentaires en cas d'humidification; par contre, si ce point se trouve au-dessous, le sol est surconsolidé et on doit s'attendre à des gonflements. 


\section{CONCLUSIONS}

L'état de non-saturation dans lequel se trouvent la plupart du temps les sols dans de nombreuses régions soulève des problèmes qui d'habitude ne sont pas traités dans la Mécanique des Sols classique, qui est axée spécialement sur des sols saturés.

La compréhension et la prévoyance du comportement des sols non-saturés supposent une connaissance assez approfondie des phénomènes d'interactions des phases constituant le sol et les autres corps poreux hydrophiles.

La succion de l'eau, qui se trouve dans les pores des sols représente l'effet résultant des forces d'interactions, exprime la capacité de rétention de l'eau par la phase solide, détermine les propriétés physiques et mécaniques et influence tous les phénomènes qui ont lieu dans ces corps hydrophiles, comme par exemple l'échange de masse (migration de l'eau) ou d'énergie (dégagement de la chaleur par mouillage, énergie nécessaire pour le drainage ou la filtration d'eau, etc.).

L'établissement de la courbe de rétention (succionteneur en eau) qui, généralement, se fait d'une manière expérimentale, peut être sensiblement facilité à l'aide des corrélations entre les teneurs en eau correspondant à différentes succions et des indices hydrauliques, comme la teneur en eau d'hygrosopicité maximale $\left(w_{\mathrm{HM}}\right)$, dont la détermination est plus simple. Dans le même but, on peut utiliser l'expression analytique que l'on a établie pour la courbe de rétention et qui conserve sa validité dans le domaine le plus intéressant (1 à 15 bars).

La courbe de rétention trouve son application pour comparer les différentes méthodes de drainage, pour estimer la distribution des teneurs en eau d'équilibre sous les revêtements imperméables et les bâtiments, pour étudier les phénomènes de migration de l'eau (infiltration, ascension capillaire, drainage, accessibilité de l'eau pour les plantes) à travers les sols nonsaturés et pour l'approche scientifique des autres questions qui intéressent le génie civil et l'agriculture.

De même, la capacité de rétention de l'eau par le sol présente un intérêt théorique dans l'étude de transferts d'énergie liés au drainage et au mouillage; processus qui, à côté de la filtration, représente les deux aspects que l'on peut distinguer pendant la migration de l'eau à travers les sols non-saturés. Ce fait donne la possibilité d'approcher les processus qui ont lieu dans les sols à l'aide des lois de la conservation de la masse et de l'énergie. Dans le cas des sols saturés, on fait d'habitude appel seulement à la loi de la conservation de la masse comme par exemple dans la théorie de la consolidation des couches argileuses.

Par contre, pour le cas plus général des sols non saturés, où il n'existe pas de relation univoque entre l'état d'humidité et l'état de serrage, on est obligé de recourir aux deux équations de bilan, celle de la masse et celle de l'énergie. Sur cette base, il sera possible à notre avis de développer à l'avenir une mécanique des sols non-saturés.

Comme l'état de serrage et de l'humidité des sols non-saturés varient dans un certain domaine pour l'étude systématique de leurs propriétés, il est recomanmandé d'utiliser l'abaque Terracina qui peut servir de base en vue d'établir des diagrammes caractéristiques de la variation de l'indice géotechnique étudié en fonction de l'état du sol. Ces sortes de diagrammes permettent une meilleure systématisation des résultats pendant l'étude des différents types des sols, un contrôle efficace des nouveaux résultats obtenus et une évaluation réaliste du comportement des sols non-saturés sous l'action des sollicitations. Cette manière de représenter l'état et d'étudier les propriétés est particulièrement utile pour les sols sous-consolidés pouvant s'affaiser par inondation (loess) et pour les sols argileux surconsolidés, capables de développer de grands gonflements et pressions.

Les problèmes présentés dans cet exposé constituent des exemples de la manière dont nous avons abordé la recherche. Nous sommes parfaitement conscients que le travail accompli constitue seulement un commencement sur la longue route qui mènera à une meilleure prévoyance et une meilleure maîtrise des phénomènes engendrés dans les sols non-saturés par l'activité humaine. 


\section{BIBLIOGRAPHIE}

[1] AITCHISON (W.E.J.M.). - « Suction profiles in soil beneath covered and uncoverered areas", 5 th, Int. Conf. on Soil Mech. and Found. Eng., vol. 2, pp. 187-191, Dunod, Paris (1961).

[2] ANDREI (S.). - Discussion, Compte rendus du $5^{e}$ Cong. Int. Méca-Sols, Paris. T. 3, pp. 113-116 (1961).

[3] ANDREI (S.). - - «e drainage de l'eau dans les sols à granulation fine ", Eyrolles, Paris (1966).

[4] ANDREI (S.). - " L'eau dans les sols nonsaturés " (en roumain). Editura Technica, Bucarest, (1967).

[5] ANDREI (S.). et Coll. - «Utilité d'étudier l'influence simultanée de l'état d'humidité et de serrage sur la caractéristique des sols nonsaturés " (en roumain). Studii, si cercetari INCERC, nr. 1, pp. 1-18 (1965).

[6] ANDREI (S.). et Coll. - «Influence de l'état d'humidité et de serrage sur les propriétés du limon de Bucarest " (en roumain), studii si cercetari INCERC, nr. 1, pp. 91-126 (1965).

[7] ANDREI (S.) et ANDREI (D.). - «Soil and rock pore water suction ", Proc. Int. Cong. of the Int. Assoc. of Eng. Geology, vol. I, pp. 272-282 (1970).

[8] ANDREI (S.) et Coll. - " Recherches concernant la sensibilité à l'humidification du loess " (en roumain). Hydrotechnica $N^{\circ}$ 6, pp. 173-187 (1962).

[9] ANDREI (S.) et PETRICA (I.). - - La détermination de la succion de l'eau des sols» (en roumain), Bu. Inst. Constr. Bucarest, $\mathrm{N}^{\circ} 3$ (1969).

[10] ANDREI (S.) et STANCU (C.). - " Influence de la teneur en eau et de la densité des sols non-saturés sur leurs caractéristiques physicomécanique » (en roumain). Hydrotechnica, № 5 , pp. 212-220-224 (1974).

[11] ANTONESCU (I.). - "Contributions à l'étude du comportement du loess » (en roumain), Thèse Institut de Construction, Bucarest (1971).

[12] CHIRITA (C.D.), ANDREI (S.) et MOISE (E.). - " Etude énergétique de l'humidité des sols dans des buts écologiques » (en roumain), dans " les sols des montagnes Bucegi ", Compte rendus de la Conférence Nationale de Pédologie, Editura Academici R.S.R. Bucarest (1969).

[13] CRONEY (D.). - « The movement and distribution of water in soils ", Géotechnique, London, Vol. 3, nr. 1, pp. 1-16 (1952).

[14] DUMBLETON (M.J.) et WEST (G.). - «The suction and strength of remolded soils as effect- ed by compression " Road Research Report LR. 206. Crawthorne (1970).

[15] KASSIFF (G.). - « Studies and design criteria for structure on expansive clays ", in Proc. Int. Res. Conf. on expansive clays, Texas A \& M University (1965).

[16] KAZARNOVSKI (B.D.). - « Evaluation de la résistance au cisaillement pour les études de routes » (en russe), Autotransizdat, Moscou (1962).

[17] KLEIN (A.). - « Method of soil testing as applied to the design of road fondations », Proc. South Africa Soc. Civ. Eng. Vol, 44 (1946).

[18] MARSHALL (T.J.). - «A relation between permeability and size distribution of pores ", Journal of Soil Science, vol. 69, $\mathrm{n}^{\circ}$ 1, pp. 1-18 (1958).

[19] MILANOVIC (D.). - « Some engineering properties of loess ", Proc. of the Speciality Session on Engineering Properties of Loess and other collapsible soils" (Mexico, 1969), pp. 11-23, publié par la Société Argentine de Mécanique des Sols, Cordoba, Argentine (1970).

[20] NASCIMENTO (U.). - « Notes sur la stabilisation des sols ", (en portugais). TechnicaReviste des Alumes do I.S.T., pp. 323-347, Lisboa (1963).

[21] PETERS (D.B.). - « Water uptake of corn roots as influenced by soil moisture content and soil moisture tension ", Soil Sci, Soc. of Am. Proc. V. 21 (1957).

[22] RUSSAM (K.), - «The mouvement and distribution of moisture beneath overseas airfields ", Road Research Board Technical Paper, No 58, London (1963).

[23] SILVEIRA (S.E.B.). - «Statistical research on stress-strain characteristics of the typical compacted soil under different moulding parameters ", Proc. 6th Int. Conf. on Soil Mech. and Foundation Eng. University of Toronto Press, Montreal, 1965, pp. 364-376. (1965).

[24] STANCULESCU (Ion). - Communication privée (1960).

[25] TERRACINA (F.). - - "Variabilité des caractéristiques d'état des sols. Représentation graphique ", Rev. Gén. Routes et Aérodromes, $\mathrm{n}^{\circ} 279$ (1958).

[26] WISEMAN (G.). et ZEITLEN (J.). - « Swelling studies on laboratory compacted clay », Technion, Haifa.

[27] WOOLTORTON (F.L.D.). - « Moisture content and CBR method of design ", Highway Special Report, n० 40 , pp. 226-252. 

